Stress and Coping

An Economic Approach

K. Wälde

Discussion Paper 2015-18

Institut de Recherches Économiques et Sociales de I'Université catholique de Louvain

IRES 


\title{
Stress and Coping - An Economic Approach
}

\author{
Klaus Wälde ${ }^{1}$ \\ Johannes-Gutenberg University Mainz and CESifo
}

October 6, 2015

\begin{abstract}
Stress is ubiquitous in society. In our model, stressors translate into subjective stress via an appraisal process. Stress reduces instantaneous utility of an individual directly and via a cognitive load argument. Coping can be functional and under the control of the individual or more automatic with dysfunctional features. We predict the occurrence and frequency of uncontrolled coping - emotional outbursts - as a function of an individual's personality and environment. Outbursts cannot always be avoided. Delaying emotional outbursts artificially can lead to even more outbursts. Looking at the effect of psychotherapy shows that expecting little and being emotional can help maximizing well-being.
\end{abstract}

JEL codes: D03, D91, I12

Keywords: Stress, coping, personality, controlled vs. automatic reaction, emotional outbursts, optimal stopping problem

\section{Introduction}

[Motivation] Stress is a feeling everybody knows. There are just those days where there are simply too many demands that fall upon oneself and it is just impossible to respond to them all. Stress is a topic that has always received a lot of attention. Whether one reads handbooks of stress written more than 30 years ago, or looks at current discussions in science and in the media, it is an amazingly and maybe disturbingly popular topic. Selye $(1982$, p. 7$)$ from a medical perspective wrote: "Nowadays, everyone seems to be talking about stress". The psychologists Lazarus and Folkman (1984, p. 1) wrote: "It is virtually impossible today to read extensively in any of the biological or social sciences without running into the term stress." Public discussion today covers various topics related to stress, such as the burnout syndrome, happiness and overall life satisfaction, the increasing use of therapeutic and medical services to cope with stress and the like. ${ }^{2}$

[The open issue] Despite the huge relevance of stress that hardly anyone would deny, an economic analysis of stress does not seem to exist. This is even more surprising as it is widely

\footnotetext{
${ }^{1}$ Contact details: Klaus Wälde, Johannes-Gutenberg University Mainz, Mainz School of Management and Economics, Jakob-Welder-Weg 4, 55131 Mainz, Germany, fax + 49.6131.39-23827, phone + 49.6131.39-20143, waelde@uni-mainz.de. I would like to thank Pierre Cahuc, Ernst Fehr, Drew Fudenberg, Johannes Haushofer, Frank Heinemann, Sendhil Mullainathan, Henning Müller, Nick Netzer, Rémi Piatek and Robert Sugden for comments and discussions and David Laibson for encouragment. Special thanks go to colleagues from psychology departments, including Brad Bushman, Andreas Eder, Boris Egloff, Laurenz Meier and Agnes Moors. The paper also gained from the CESifo behavioural economics conference and from seminar presentations at, inter alia, Aarhus University, Humboldt University, NYU, Polytechnique Paris and TU Vienna.

${ }^{2}$ A German governmental agency issued a stress report (Lohmann-Haislah, 2012) and a major health insurance company put a special emphasis on stress in its annual report (DAK, 2014). The French government issued a report on happiness (Stiglitz, Sen and Fitoussi, 2008) and Helliwell, Layard and Sachs (2013) edited a world happiness report. Both of these reports are full of references regarding the concept of stress. The OECD (2014), more generally speaking, argues that mental illness poses a large economic burden on society, despite rising expenditure on mental health.
} 
accepted that many economic events generate large numbers of stressors. Biased technological change, distributional effects caused by globalization, unemployment or the great recession are undeniably (at least at a subjective level) related to the stress of individuals. What is missing in economics is a concept of stress that can be integrated into standard economic analyses. ${ }^{3}$

[The objectives] The first and foremost objective of this paper is, therefore, to provide a formal framework within which to think about stress and its implications. This approach follows "a desire to expose how <existing views> are wrong" (all quotes in this paragraph are from Rabin, 2013a) or, to put it a bit more positively, to work out what existing views actually mean. Theories developed in most social sciences are what could be called "imprecise theories". Models of stress and coping developed in other disciplines lack mathematical rigor and precision. This makes it "just harder to identify flaws". Translating standard psychological views into an otherwise mainstream economic model allows us to "see their limits that can guide us in further improvements". To make the point most clearly: What is a stressor, what is stress, what is coping once these concepts are cast into a formal framework? ${ }^{4}$

[The setup] The model in section 2 represents stress as a subjective feeling captured by a stochastic state variable which increases in the presence of stressful events (stressors), and falls via coping measures and in the presence of pleasant events. The process starting with the stressor and ending with stress is an example of what psychologists call an appraisal process, i.e. the evaluation of events "with respect to their implications for well-being" for the person facing the stressors (Lazarus, 1999, p. 75 ). ${ }^{5}$ Stressors are kept exogenous in our setup and appraisal is modelled by certain functional forms of stressors. Coping measures are endogenous and (at least partially) optimally chosen by the individual. The effects of stressful or pleasant events and of coping measures on stress depend on the personality of the person under consideration. ${ }^{6}$

Starting with stressors and following a distinction made in psychology, we distinguish between events that occur relatively often and others that occur more rarely. Historically, the more rare events were seen as the classic sources of stress. A widely cited list of stressors was compiled by Holmes and Rahe (1967) in their Social Readjustment Rating Scale (SRRS). This scale includes events (in descending order of stressfulness) such as 'death of spouse', 'divorce', 'jail term', 'fired at work' and so on, concluding with 'vacation, 'Christmas' and 'minor violations of law'. It was later discovered that, even in the absence of any of the 43 events listed in the SRRS, individuals can be under high stress given "daily hassles" (Kanner et al., 1981) such as 'losing things', 'don't like fellow workers', 'too many obligations' and the like. Our specification for the evolution of stress allows for both types of events, reflecting the fact that empirically both of them matter. ${ }^{7}$

When modelling the effect of a stressor on the stress level of an individual, we allow for intermediate cognitive processes of the individual experiencing a certain stimulus and displaying the response (Lazarus, 1993). These appraisal processes are captured in our setup by parameters which describe how a stressor translates into stress.

\footnotetext{
${ }^{3}$ There are some empirical analyses using measures of stress to understand the impact on birth weight (Camacho, 2008), to understand the link between parental stress and child outcomes (Baker et al. 2008), the effect of housing vouchers on mental health (Kling et al. 2007) and the gender differences in abilities to cope with stress (Kling et al. 2005). The theoretical analysis by Caplin and Leahy (2001) use stressful events as an example of how anxiety can be built up (e.g. a medical procedure, see Caplin and Leahy, 2004). Yaniv (1995) presents a static model of worker health where the firm takes the effects of a stressful environment into account. None of these analyses studies the evolution of stress over time or the role of appraisal and coping.

${ }^{4}$ The technical answer in chronological order: an exogenous parameter, a state variable and a control variable.

${ }^{5}$ See the main part and footnote 19 for more elaboration and references on appraisal theory.

${ }^{6}$ As will be discussed in the main text, the personality measures used here are "deeper" measures of personality than measures such as the "Big 5". For the background in psychology, see John et al. (2008). Applications in economics are discussed, for example, by Borghans et al. (2009).

${ }^{7}$ To be fair, there are many "little things in life" that can reduce stress. In fact, Kanner et al. (1981) also inquire about "uplifts" in their questionaire.
} 
The channel through which stress is detrimental to an individual's utility is motivated by the literature on cognitive load. ${ }^{8}$ This literature builds on the view that cognitive processes can best be understood by using the concept of a working memory. ${ }^{9}$ This working memory has a certain capacity for processing information. Stress, anxiety or other strong feelings imply cognitive load which reduces the amount of resources available for other information processing. To make a simple example, stress reduces the ability to work intensively or focus on other important activities. In fact, we will model the negative effects of stress on individual wellbeing by modelling the performance-reducing effect of stress. Stress reduces effective labour supply at work.

Turning to coping strategies, standard classifications distinguish between "emotion focused" copying strategy as opposed to "problem focused" behaviour, which addresses the stressor itself (Lazarus and Folkman, 1984, ch. 6). ${ }^{10}$ Other classic categories include "functional" vs. "dysfunctional" approaches, whereas modern views of coping often include a distinction between "automatic" and "controlled" processes (e.g. Connor-Smith et al. 2000, Skinner and ZimmerGembeck, 2007). ${ }^{11}$

Coping behaviour in our model can easily be understood using these categories. The feature of coping we emphasize is its stress-reducing nature. We, therefore, model an "emotion focused" coping strategy chosen by an individual that reacts optimally to subjective stress levels. This strategy reduces stress in a gradual and deterministic fashion. This is a "controlled" process. At the same time, we allow the individual to be overwhelmed by stress experiences. Being overwhelmed triggers behaviour that can be understood as uncontrolled and that leads to a discrete and fast reduction of stress. This reflects the "automatic" process of the above categorization. As examples for the smooth coping style, think of 'sorting things out', 'talking to a friend' or 'doing sports'. The more violent and less controlled behaviour stands for 'emotional outbursts' taking the form of yelling, making gestures while driving the car, getting drunk, becoming aggressive and the like.

Section 3 models individual behaviour as an optimal stopping problem. ${ }^{12}$ Stress evolves continuously with discrete jumps at random points in time and is controlled by smooth coping. Discrete increases and decreases are caused by negative or positive surprises. ${ }^{13}$ When the stress level is too high, an emotional outburst occurs. The outburst reduces the stress level by a discrete amount. Smooth coping allows the reduction of stress in a continuous way. Coping is optimally chosen and takes the effect of random surprises and outbursts into account.

[Findings] Given optimal behaviour, stress evolves smoothly most of the time, but is occasionally disrupted by surprises and outbursts. Section 4 finds that coping behaviour depends crucially on the interplay between the situation (stressors) and the individual's personality.

\footnotetext{
${ }^{8}$ This literature consists of various relatively independent substrands. An introduction from the perspective of learning is provided by Paas et al. (2003). A recent contribution to the understanding of the effect of cognitive load on performance is by Hoffmann et al. (2013). The role of cognitive load in the context of anxiety and performance is surveyed in Derakshan and Eysenck (2009).

${ }^{9}$ The view goes back to Baddeley and Hitch (1974). See, for example, Smith and Kosslyn (2007, ch. 6) for an introduction.

${ }^{10}$ Economics is full of problem-focused coping styles. An unemployed worker putting effort into searching for a job would be one such example.

${ }^{11}$ This follows the tradition of dual-process models used in psychology more widely. See Chaiken and Trope (1999) for an overview and Strack and Deutsch (2004) or Sherman et al. (2008) for more recent contributions.

${ }^{12}$ Optimal stopping features arise as emotional outbursts imply fixed utility costs in our setup. In this sense, the paper is related to, for example, Bentolila and Bertola (1990), Bertola and Caballero (1994) and Dixit (1989). See Stokey (2008) for an excellent introduction. Optimal stopping problems in the presence of Poisson uncertainty (as in the present analysis) is treated in Dixit and Pindyck (1994 ch 5.B) and was used by Ahlin and Shintani (2007) or Chen and Funke (2005).

${ }^{13}$ Surprises occur when the expected outcome of some event differs from the subjective expectation of an individual with respect to this outcome. This is related to the disappointment literature of Loomes and Sugden (1986), Bell (1985) and others.
} 
While a strong negative surprise can induce any individual to display uncontrolled coping styles, our setup suggests the classification of individuals into "stress-prone" and "stress-resistant" categories. Stress-resistant individuals never display emotional outbursts in the absence of negative surprises. Stress-prone individuals can display emotional outbursts even when there are no negative surprises. Daily hassles alone can lead to overwhelming stress experiences. Yet, this is not necessarily the case. Even stress-prone individuals can be "good stabilizers", i.e. they can experience reducing stress levels over time. This is conditional on their initial stress level. If the latter is too high, they turn into "bad stabilizers" and will foreseeably display an emotional outburst at some deterministically given future point in time. Due to this dependence of behaviour on the current stress level, stress-prone individuals display more volatile behaviour than stress-resistant individuals.

An outburst theorem will then relate situational and personality parameters to allow a prediction under which conditions and outburst will occur. The "growth rate of stress", the individual's tolerance level (above which outbursts occur) and the current stress level all play a role.

[Applications] Based on these general findings, we use our framework to study various issues that commonly arise in a context of uncontrolled and aggressive behaviour. We first ask what would happen if individuals could control their tolerance level. Surprisingly, we find that outbursts occurring early, i.e. at low stress-levels, have higher chances of permanently reducing the stress level of an individual than emotional outbursts that take place only later. Suppressing outbursts is, therefore, not a good idea. We thereby provide a rationale for common recommendations to "not bottle one's anger up inside" or to allow for "air-cleaning" angry outbursts (Bushman et al., 2001).

Second, we compute the frequency with which outbursts occur when a bad stabilizer is caught in an "outburst cycle". We find that a higher growth rate of stress and a higher tolerance level lead to a higher frequency of outbursts. This also points to the fact that high tolerance levels might not be a desirable characteristic. We also find that the intensity of an outburst, i.e. the amount by which stress is reduced as a consequence of the outburst, has ambiguous effects on the frequency of outbursts. One could believe that a highly intense outburst is desirable, i.e. it would decrease the frequency of outbursts. At the same time, however, when outbursts reduce stress by a large amount, the individual will anticipate this and reduce costly smooth stress-reduction activities. This, in turn, increases the frequency of outbursts.

Third, we ask how individuals react to positive and negative surprises. It again turns out that the distinction between stress-prone and stress-resistant individuals is crucial. Stressresistant individuals react to temporary shocks in a transient way. A negative surprise increases the stress level, which then gradually falls over time to return to "normal" levels again. A stressprone individual, however, can permanently suffer from a negative shock. While experiencing low stress levels and displaying correspondingly smooth stress-reduction behaviour before the negative surprise, the surprise can push this individual into an outburst cycle. Leaving this outburst cycle then requires major interventions: Either a positive surprise is experienced or daily stressors are reduced to a minimum such that the individual has a chance to gradually reduce stress levels. This explains the large heterogeneity observed in individual behaviour after major stressful events. While some people return to normal "as if nothing has happened", others persistently suffer from a temporary event.

Our next application, a first step towards a "theory of therapy", departs from one of the most fundamental building blocks in economics and also from (parts of) personality psychology. By contrast, this application is very much in line with clinical psychology and therapy studies more generally speaking. We ask what would happen if individuals could change their tastes and attitudes. There are powerful arguments in economics that tastes should be regarded as stable and maybe even identical across individuals (Stigler and Becker, 1977). By contrast, 
more modern views argue that preferences are influenced by social circumstances (Fehr and Hoff, 2011) and are therefore endogenous. Personality psychologists also seem to be moving from the more traditional view of stable personalities to personalities that change over time (e.g. Specht et al., 2011), a fact also stressed by Borghans et al. (2008). Clinical psychology has always stressed the importance of reappraisal of certain outcomes (stressors in our application) to improve emotional stability (e.g. Allen et al, 2008, p. 230). We model this clinical psychology approach by inquiring into the effects of changes in personality parameters. If individuals were able (say, by therapy) to change their personality, how would they want to do this? ${ }^{14}$

Our final application is more "forward-looking" and goes beyond our analysis of stress and coping. Our paper suggests model-consistent definitions of personality characteristics (e.g. stress-prone vs. stress-resistant individuals). These characteristics are well-defined functions of personality parameters. Based on modern factor analysis (see e.g. Heckman et al., 2006 or Conti et al., 2014), we will discuss how these parameters can be structurally estimated. An interpretation of personality characteristics would then be much easier and more disciplined (by our theoretical model) than personality measures that are based on purely statistical methods. Future empirical work would have to show how our theoretically grounded personality measures can be estimated from answers to standard questions in commonly available questionnaires.

[Related literature] In spirit, we see our paper in the tradition of the strand of behavioural economics that uses ideas from psychological research to better understand human behaviour. One can trace back the formal tradition at least to Strotz (1956) on time-inconsistencies, with the more recent new interest inspired by Laibson (1997). ${ }^{15}$ Our interest in stress places our analysis into the modern literature analysing emotions from an economic perspective. Economic analyses of emotions include ex-post emotions such as regret, disappointment and rejoicing (Loomes and Sugden, 1982, 1986, Gul, 1991), ex-ante emotions such as joy and fear (Caplin and Leahy, 2001, 2004, Köszegi, 2006, Becker and Rubinstein, 2011) and immediate emotions or visceral factors. Examples are desire and compulsion (Laibson, 2001, Bernheim and Rangel, 2004 and Brocas and Carrillo, 2008) or hunger and thirst (Loewenstein, 2000). A final group consists of belief-based emotions either in psychological games (Geanakoplos et al., 1989), for which an application studies guilt in marriage (Dufwenberg, 2002), or in non-strategic settings as in Compte and Postlewaite (2004), who analyse self-confidence. See Wälde (2015) for a more extensive review of emotion research in economics.

Given the behavioural predictions of our analysis, the paper is also related and generalizes analyses based on cues, dual-self views of human decision making or on time-inconsistent behaviour. In some broad interpretation, these models allow to understand behaviour à la Dr. Jekyll and Mr. Hyde: One and the same individual sometimes displays very reasonable, rational and relaxed behaviour while, at other times, the individual acts as if it was another person. Where does this apparently inconsistent behaviour of individuals come from? ${ }^{16}$

Laibson's cue theory (2001) explains how cue-dependent preferences allow the understanding of such an erratic behaviour. Impulsive behaviour of individuals can be understood by cues affecting their preferences (without touching meta-preferences, which are stable). Accumulated cues can induce an individual's marginal utility from consumption to go up rather quickly and strongly. Such a sudden "craving" for a certain good leads to behaviour that is very different from situations displaying no or other cues. In the framework of Bernheim and Rangel (2004), individuals are sometimes in a "cold" mode where decision making about consumption behaviour is rational. When triggered by some cue (whose probability to occur depends on

\footnotetext{
${ }^{14}$ I am grateful to Ernst Fehr for having brought up this question.

${ }^{15}$ The link between psychology and economics is, of course, much older, starting at least with Bentham's (1789, 1970, p. 11) "two souvereign masters, pain and pleasure". For a revival of the link between subjective well-being (happiness) and choice, see inter alia Benjamin et al. (2012) or Rayo and Becker (2007) and Kahneman, Wakker and Sarin (1997). A short history of bounded-rationality models is provided by Harstad and Selten (2013).

${ }^{16}$ I am grateful to Nick Netzer for suggesting this perspective and for discussions of these aspects.
} 
current and past decisions of the individual), individuals are in a "hot" mode and act against their preferences.

In dual-self models, two selves are in conflict with each other and play a game over a certain behaviour (Fudenberg and Levine, 2006, 2012). This structure can explain many empirical regularities observed in the context of self-control problems. A central component of these models are costs of self-control. When they are high, there can be procrastination and delay (as in O'Donoghue and Rabin, 1999, 2001). High self-control costs also reduce the saving rate of an individual. By contrast, different behaviour in the sense of higher saving rates can be observed when self-control costs are lower, e.g. when cognitive load is lower. Intrapersonal tension in the principal-agent setup of Brocas and Carrillo (2008) is modeled for the case of temptations by a discrepancy between one utility function capturing the "true instantaneous payoff" (principal) and another utility function describing the individual's motivation (agent). When there is incomplete information, behaviour of the individual either follows a smooth consumption rule for the tempting good that maximizes motivational utility or it is restricted by a consumption cap. These differences in behaviour can result from changes in the valuation of the tempting good or from changes in relative prices or endowment.

In models of time-inconsistent behaviour, we can also see abrupt changes in behaviour. In Strotz' (1956) continuous-time analysis, an individual can be argued to follow a plan in some smooth way up to the point where he reconsiders the plan and readjusts his behaviour in a discrete way (see his figure 2). In discrete time setups (Laibson, 1997), an individual makes a plan just to realize one period later that $\mathrm{s} /$ he should not follow this plan (unless $\mathrm{s} /$ he is a sophisticated time-inconsistent person as in O'Donoghue and Rabin, 1999). In the absence of any self-commitment devices (Fudenberg and Levine, 2006, 2012, Ali, 2011), any revision of early plans leads to a strong and sudden change in observable behaviour.

In our setup, we also see two apparently inconsistent behaviours of individuals. At points, an individual appears very calm and "reasonable", at other points, the individual acts in an aggressive way. However, we do not base this upon random cues, changes in parameters, cognitive load or revisions of decisions. Behaviour of individuals is rather the outcome of an accumulation of feelings resulting from various previous events. Smooth and controlled behaviour can endogenously be followed by less-desirable outbursts and vice versa (see, for example, the outburst cycles below) even in the absence of any external events. ${ }^{17}$

\section{The model}

\subsection{Origins of stress}

Emotional tension (which we use interchangeably with stress) can be caused by a multitude of events. Following a classic distinction in psychology, we distinguish between two types of events. Those that occur only rarely and those that occur more frequently, say daily. The stressfulness of rare events can be measured by the Social Readjustment Rating Scale (Holmes and Rahe, 1967, Hobson et al., 1998). In decreasing order of stressfulness, rare events start from 'death of spouse', 'divorce', 'jail term', 'fired at work' and end with 'vacation, 'Christmas' and 'minor violations of law'. More frequent events can be called 'daily hassles and uplifts' in the tradition of Kanner et al. (1981) and include 'losing things', 'don't like fellow workers, 'too many obligations' and others.

\footnotetext{
${ }^{17}$ Our economic approach to stress also contributes to the psychological literature. The literature on emotion regulation (see, for example, Gross, 1998, 2008, Gross and Feldman-Barrett, 2011) has considerably enlarged the earlier findings by building on and extending Lazarus' work. According to Gross (2008, p. 505), “one intriguing puzzle is why people use one emotion regulation strategy rather than another". Our model provides standard conditions (relative costs and benefits) under which one or the other regulation strategy is chosen.
} 
Our model describes the randomness of the rare events by a Poisson process with a random jump level $h(t)$ and an exogenous arrival rate $\lambda$. The jump $h(t)$ can be positive or negative according to some well-defined distribution. Our individual forms expectations about the jump level. This expected level $\mu$ can be a subjective measure or the mathematically correct mean. The surprise $g(t)$ implied by a rare event that occurs at $t$ is then given by

$$
g(t) \equiv h(t)-\mu \text {. }
$$

In the spirit of Bell (1985), Loomes and Sugden (1986) and others, we call a positive surprise, defined by $g(t)>0$, elation and a negative surprise disappointment. Both elation and disappointment are measured relative to the mean $\mu$. We consider the realization to be an exogenous and objective quantity, while the expected value $\mu$ is a personality measure of the person under consideration. $^{18}$

As an example, let an increment of the Poisson process denote the point in time when a paper is accepted for publication. Let further $h(t)$ represent the quality of the accepting journal or the number of citations a paper gets, say, within the first two years after publication. When the quality or the number is very high and the subjective expectation is modest (i.e. $\mu$ is not too high), the individual is positively surprised.

In addition to this rare source for emotional tension, there is a daily flow of events that has an impact on a person. These events imply certain demands $p(t)$, the stressors, which have to be met given the abilities $a(t)$ of the individual. When 'hassles' dominate relative to 'uplifts', $p(t)$ is positive. Just as with surprises, let us assume that pressure and abilities are objective quantities. We call $p(t) / a(t)$ the intensity of the stressor $p(t)$.

As an example, consider $p(t)$ to represent the number of emails an individual receives per day. If the ability $a(t)$ to reply to emails is high, the intensity of the stressor 'email' is low. If an individual is not that good at communicating by email, the intensity of the stressor email is high.

We model the effect of stressors on emotional tension by taking a clear causal view. Stressors cause (a change of) emotional tension. The mechanism through which stressors increase emotional tension follows "appraisal theory" where "emotions are elicited by evaluations ( $a p$ praisals) of events and situations" (Roseman and Smith, 2001, p. 3). ${ }^{19}$ The appraisal process is strongly influenced by personal characteristics of the person under consideration. Support for this modelling choice comes from appraisal analyses that take individual heterogeneity into account (for a discussion of the related aspects, see e.g. Kuppens et al. (2008), Scherer and Brosch (2009) and Tong (2010) or from the literature that looks at the cognition-emotion relation focusing on personality or clinical aspects (see Lewis, 2005, p. 171 for a short overview). We capture this appraisal process by individual-specific functions $F(p(t) / a(t),$.$) and G(g(t),$. that specify how the intensity of daily hassles or rare events influence the evolution of stress.

There is systematic empirical evidence from field experiments on the stress-reduction effect of positive surprises. Haushofer and Shapiro (2013) study the effect of random unconditional cash transfers of around $\$ 400$ or $\$ 1500$ on households in Kenya. They find that for the large transfer, there was a significant reduction of the households' stress level. ${ }^{20}$ Haushofer and

\footnotetext{
${ }^{18}$ One can think about $\mu$ as a reference point (Kahneman and Tversky, 1979) of our individual indicating what she finds "appropriate" concerning the outcome of $h(t)$. Such a reference point could be made endogenous as in Gul (1991) or Köszegi and Rabin (2006). See prop. 5 in the section on therapy below for further elaboration of the personality view.

${ }^{19}$ Appraisal is a highly complex process which we do not model in all details. Ellsworth and Scherer (2003) provide a list of dimensions along which individuals evaluate a certain event. For a full-scale summary of appraisal theory, see Scherer et al. (2001), a recent analysis of the role of appraisal in emotion is in Moors and Scherer (2012). A comparison to alternative views is e.g. in Roseman and Smith (2001) or Lewis (2005, p. 170).

${ }^{20}$ While households receiving the $\$ 400$ transfer had a lower stress level as well, it was not significantly lower relative to the treatment group receiving no transfer. This could be due to expectations $\mu$ rising immediately when a representative of a foreign NGO contacts an inhabitant of a rural village in a poor country.
} 
Fehr (2014) summarize several further studies concluding that "cash transfers reduce distress" (p. 864). If we are willing to consider random positive cash transfers as positive surprises, this is strong systematic support for our setup. Evidence for stress-increasing effects of negative surprises is just as strong (see again Haushofer and Fehr, 2014, and the studies reported therein): Whether one looks at bad weather conditions for farmers in Kenya or job-loss for Swedish workers, these events all lead to an increase in the stress levels of individuals.

\subsection{The effect of stress on individual well-being}

What are the channels through which stress affects well-being of an individual? Whether looking at semi-popular sources (e.g. www.stress.org/stress-effects) or at various scientific literatures, there are long lists of how stress reduces one's general well-being. Stressed individuals enjoy food less, play less with kids, no longer enjoy or therefore skip playing soccer and so on. There are also various bodily symptoms (like headache, dizziness, sweating, sleeplessness and other). We capture these direct negative effects by letting stress negatively affect the utility level of an individual. ${ }^{21}$

There is a lot of evidence, however, that stress also affects the individual in more sophisticated, yet not less important, ways. One of these indirect channels is the effect of stress on performance via cognitive load. The argument is simple: We start from the general view of human mental processes (see Smith and Kosslyn, 2007, esp. ch. 6 as a starting point) that an individual is endowed with a certain amount of working memory $M$. This working memory can be used for various purposes of which some are chosen consciously by an individual while others are more automatically determined. In our setup, the appraisal process evoked by stressors is one such example of an automatic, exogenous, allocation of working memory resources. As stressors are exogenous to the individual, the amount of working memory used for the appraisal process is exogenous as well.

Generally speaking, one can distinguish between low-level and high-level appraisal. Lowlevel appraisal tends to be more automatic and preattentive while high-level appraisal requires attentional and working memory resources (Kalisch et al., 2006). Most of the examples for 'daily hassles' or 'rare events' clearly require high-level appraisal. Losing the car key on the way towards an important meeting requiring a one-hour drive requires weighing various options from 'not showing up' to 'going by taxi'. Similar working-memory intensive considerations are required after rare events like 'divorce' and even 'Christmas' requires lots of thinking. We therefore capture this pre-emption of the working memory by stressors by assuming that the working memory capacity required by the individual for appraisal is given by $M(W)$, where $M^{\prime}(W)>0$, i.e. the higher the stress level $W$, the more costly appraisal processes are.

To understand the effect on performance, one then simply needs to complete the working memory constraint: What is left of the working memory can be used for everyday activities like, taking the example of a performance measure, putting effort into work, $M(e)$. The memory resource constraint of our individual therefore reads

$$
M(W)+M(e)=M
$$

Returning to consumption and income of our individual, this constraint implies that consumption is a falling function of stress. Equating consumption with income, $c=w l(e)$, where $w$ is the wage rate and $l(e)$ is effective labour supply as a function of effort $e$, consumption

\footnotetext{
${ }^{21}$ Following the concepts of experienced vs. decision utility (see e.g. Loomes and Sugden, 1982, 1986 or Kahneman et al., 1997), we are in the Bentham-tradition and allow non-observables to enter the utility function describing hedonic experience of an individual. For a broad and thorough discussion of this distinction and many other aspects concerning current economic methodology, see Caplin and Schotter (2008).
} 
rises in effort. As effort falls in stress, consumption is a falling function of stress, $c=c(W) .{ }^{22}$ If we are willing to make a simple assumption between memory and effort in (2), i.e. if we assume $M(e)=e^{1 / \varepsilon}$ with $\varepsilon>0$, the amount of effort that can be provided amounts to $e=(M-M(W))^{\varepsilon}$. Consumption is then given by

$$
c=w l\left([M-M(W)]^{\varepsilon}\right)
$$

To summarize this cognitive load idea in a very simple way: performance at work goes down when an individual is kept busy thinking about many other non-job related issues, i.e. when the individual just finds it hard to focus. ${ }^{23}$

Both the direct level and the indirect level of stress suggest that the instantaneous utility function is a function of the consumption level and of the level of stress. We denote it by $u(c, W)$ and let it rise in consumption and fall in stress.

Before continuing, let us note that there is a huge literature on cognitive load in psychology, in experimental economics and elsewhere which strongly supports our modelling approach. In psychology, Sweller (1988) is the classic reference for cognitive load theory and learning (see Paas et al., 2003 for a more recent review). According to these findings, cognitive load reduces learning. Eysenck and Calvo (1992) in their 'processing efficiency theory' and later in their 'attentional control theory' (Eysenck et al., 2007) argue that the primary effect of anxiety in stressful situations is "to pre-empt some of the processing and storage resources of the working memory system" (Eysenck and Calvo, 1992, p. 415). Hoffmann et al. (2013) provide a short literature overview in their introduction showing that accuracy of judgements generally falls under cognitive load..$^{24}$

In economics, cognitive load is modeled by Fudenberg and Levine (2006) as a parameter for the cost of self control in their dual-self model. One of their motivations is the self-control experiment by Shiv and Fedorikhin (1999) that show that individuals have a higher tendency to choose a chocolate cake rather than a fruit salad when they have to perform cognitively demanding tasks like remembering seven-digit (rather than two-digit) numbers. In terms of our mental resource constraint (2) remembering seven-digit numbers takes up more memory resources $(M(W)$ is higher) as when remembering two-digit numbers. As a consequence, resources $M(e)$ left for putting effort into self-control (i.e. choosing the healthy desert) are reduced.

Our approach also shares the idea of an additional resource constraint in human decision making with other analyses, even though the modelling is different. Banerjee and Mullainathan (2008) allow individuals to allocate one unit of attention to activities at home and at work in a development context. This choice of allocating attention is made optimally and therefore differs from the idea here that a certain amount of resources is taken up automatically by whatever the stress level happens to be. ${ }^{25}$ Fudenberg and Levine (2012) use the concept of costly cognitive resources to obtain convex costs of cognitive control. Cognitive resources are a stock ("willpower") in their setup, however, which can also become negative. This differs from our resource constraint where a stock $M$ of mental resources is available at each instant.

\footnotetext{
${ }^{22}$ This is the income-reducing effect of stress on consumption. There can clearly be a consumption-increasing effect as utility from consumption can compensate for disutility from stress. Extending this setup by allowing for savings would imply precautionary saving behaviour that would smooth consumption and would lead to self insurance against income fluctuations induced by stress.

${ }^{23}$ To make a positive example, think about adolescents or young adults falling in love. They should focus on their education, study hard for exams or apply for jobs. Being in love just takes up all of their working memory and no resources are left for thinking about anything else.

${ }^{24}$ To be fair, the point of Eysenck and Calvo (1992) and Hoffmann et al. (2013) is that performance can under specific circumstances actually increase under cognitive load as either motivation rises in stressful situations (which increases $M$ ) or individuals switch to less resource-consuming strategies. We believe that these circumstances are more the exception than the rule.

${ }^{25}$ Limited resources are also taken into account by Mullainathan (2002) who stresses the limited capacity of a human's memory.
} 
The empirical findings of Mani et al. (2012) also strongly support our modelling approach. They find that cognitive performance is lower for poor farmers. This can be explained partially by poor farmers being more stressed (measured by heart rate and blood pressure). This is perfectly in line with a cognitive load argument as employed here. ${ }^{26}$

\subsection{Coping with stress}

Coping styles can be ranked according to how functional vs. dysfunctional they are. Frequent strategies ranked in this way start with 'problem solving' and 'support seeking', continue via 'emotional expression' and 'aggression' to end up with 'wishful thinking' and 'worry' (Skinner et al., 2003). Another categorization distinguishes between problem-focused and emotion-focused copying styles (Lazarus and Folkman, 1984). A final important distinction central for our approach distinguishes between automatic and controlled processes (e.g. Connor-Smith et al. 2000, Skinner and Zimmer-Gembeck, 2007).

We will model coping strategies here which are controlled and emotion-focused and which also feature some automatic and dysfunctional properties. A standard example for controlled emotion-focused coping activities is communication. Individuals can talk to friends, relatives or, more systematically, to a therapist. Another example is practicing sports, especially endurance sports which strongly reduces stress hormones. We will denote controlled coping activities below by $m(t)$. It will be the outcome of utility maximization as described further below.

A less controlled emotion-focused coping activity is what we call an 'emotional outburst'. Outbursts occur when individuals feel overwhelmed by a situation, when emotional tension is too high and individuals can't help but "explode". This can take the form of honking while driving the car, swearing when sitting in the office, banging one's fist on the table or other. It is a relatively short event (say, less than 5 seconds). This approach is dysfunctional in the sense that it might be bad for the individual's environment or detrimental for the reputation of the perpetrator. This will be modeled further below by fixed utility costs. At the same time, however, emotional outbursts also have a functional side as they reduce tension. Denoting the instant before an emotional outburst by $\tau_{i-}$, the effect of an outburst is to reduce tension by a fixed amount $\Delta>0$,

$$
W\left(\tau_{i}\right)=W\left(\tau_{i-}\right)-\Delta
$$

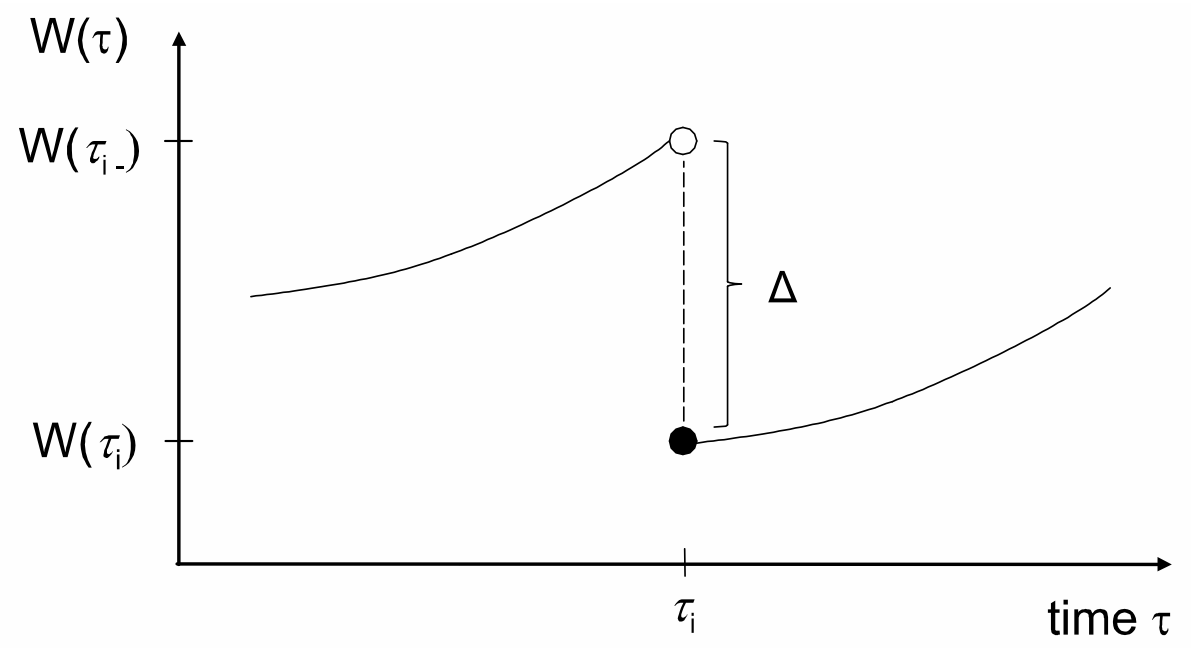

Figure 1 The effect of an emotional outburst on stress $W(t)$

\footnotetext{
${ }^{26}$ Clearly, stress does not fully explain variations in cognitive performance in Mani et al. (2013). Poverty per se causally captures attention and thereby also reduces cognitive resources. See Mullainathan and Shafir (2013) for the broader perspective.
} 
Is there evidence that supports such a "stress-reduction technology" as illustrated in fig. 1? One could see $\Delta$ as an example of the ancient idea of "catharsis", a quick purification from overwhelming feelings. There are many metaphors that suggest so. In everyday life, people in angry situations often suggest to themselves and others to "let it out", the recommendation "do not bottle your anger up inside" is well-known and the idea of a "air-cleaning" angry outbursts is common. Bushman et al. (2001), from where these examples were taken, follow this intuition and device experiments that show that people reacted more aggressively when they believe that outbursts reduce their anger. In terms of (4), people who believe that stress reduces from $W\left(\tau_{i-}\right)$ to $W\left(\tau_{i}\right)$ by an outburst, choose a larger $\Delta .^{27}$

There is further evidence for a positive catharsis effect coming from self-reports of individuals perpetrating psychological aggression as defined by the 'conflict tactics scale' (see table 3 of Straus et al., 1996) where psychological aggression includes insulting, swearing, shouting, stomping out of the room and other. Shorey at al. (2012) report very heterogeneous results showing that $42.6 \%$ of respondents "felt less angry" while $41.7 \%$ felt more angry. Some of the perpetrators $(25.7 \%)$ felt less stressed, $40.2 \%$ felt more stressed. When one computes the average effect of these outcomes (adding up the level of changes times the share of individuals reporting this change), individuals clearly reduced stress or anger.

\subsection{The dynamics of stress}

We are now in a position to summarize the various components of a stressor-appraisal-stresscoping episode in an equation that describes the dynamics of stress. We let emotional tension $W(t)$ follow a stochastic differential equation that reads

$$
d W(t)=\left\{F\left(\frac{p(t)}{a(t)}, W(t)\right)-\delta(m(t), W(t))\right\} d t+G(g(t), W(t)) d q(t) .
$$

The first term captures the effect of demand relative to ability, $p(t) / a(t)$. A classic definition (Lazarus and Folkman, 1984) that was adopted by numerous authors thereafter defines stress as the mismatch between demands of a task and the abilities of a person to cope with those demands. Our approach follows this definition in spirit but goes beyond it by adopting a dynamic perspective. The stressor intensity $p(t) / a(t)$ is a source of a change of tension, not of tension itself. Tension is a subjective feeling that can increase and decrease over time. Only in exceptional cases does it change in a discrete way. The views of Lazarus and others sound more static which would imply that any tension level, any stress is gone once the stressor is gone. Here, tension persists beyond the point where the cause of tension, the stressor, was present. Tension can be reduced subsequently.

\footnotetext{
${ }^{27}$ Common wisdom in social psychology seems to be that "catharsis does not work", i.e. $\Delta$ would have to be negative in our model. The modern experimental evidence supporting this claim often combines two simultaneous interventions. One is a "pure" venting intervention like hitting a punching bag and the other is a rumination intervention (Bushman, 2002). In another setup (Bushman et al., 1999, study 2, p. 371, right column), participants were reminded of the person who had made them angry before hitting the punching bag. As Bushman (2002) writes, cognitive neoassociation theory would suggest that aggressive thoughts activate further aggressive thoughts which are linked to each other in an associative network. Rumination and thinking of the other person therefore makes individuals more aggressive. Even if the pure venting intervention of boxing had a anger-reducing effect, the negative association effect would more than overcompensate the boxing effect. As a conclusion (and leaving aside considerations on causality in setups with self-selection), venting of anger makes individuals more angry.

The big difference we see to our idea of an outburst is that outbursts are spontaneous acts and not a deliberate activity. In terms of the general aggression model of Anderson and Bushman (2002, fig. 2), we see outbursts as an impulsive action and smooth coping as a thoughtful action. In all catharsis experiments, participants are willingly performing some act (e.g. hitting). They never display some automatic reaction. Measurements from these experiments should therefore not be used to fix the sign or magnitude of $\Delta$. I am grateful to Brad Bushman for comments on this issue.
} 
Individual ability is not the only individual characteristic which matters for how a stressor affects an individual. The intensity of a stressor, given a certain current tension level $W(t)$, is an argument of some increasing function $F($.$) . This function captures personality differences$ beyond differences in ability. One and the same amount of relative pressure for one and the same tension level $W(t)$ will affect one individual more than another. For two individuals $i$ and $j$, we allow that $F_{i}(p(t) / a(t), W(t))>F_{j}(p(t) / a(t), W(t))$. Note that there is strong evidence for this view. Meier et al. (2006) study the reaction of individuals to aggression-related cues. Individuals with low measures of agreeableness (one of the Big 5 personality measures) reacted considerably less aggressive than the average individual.

The second term $\delta(m(t), W(t))$ in the deterministic part of (5) is a depreciation function and captures the effect of automatic and intentional reduction in tension. Automatic reduction takes place as there is some natural tendency in humans to "cool down" over time. Absent new stressors and given a historically given stress levels, one should expect that emotional tension $W(t)$ stabilizes over time to end up at some constant level. We call this mechanism the "autonomous stress reduction potential" of a person. Intentional reduction of emotional tension results from coping activities $m(t)$. This is a control variable of the individual and will be optimally chosen further below.

While the first and second term affect emotional tension in a deterministic and foreseeable way and thereby captures common everyday events, surprises come at random points in time. The arrival of (potential) surprises is governed by a Poisson process $q(t)$ with a given arrival rate $\lambda$. As explained after (1), the surprise $g(t)$ can be positive or negative. Given the current tension level $W(t)$, a positive surprise translates into less tension. By how much tension reduces given a positive surprise depends on personal characteristics. Likewise with negative surprises. Some individuals have somatic responses (like heart attacks in extreme cases) while others just seem to able to "ignore" negative surprises. These characteristics are captured in the function $G($.$) . Whatever the state of the world, it is an individual's evaluation G($.$) that determines$ how he is affected by this state.

We define emotional tension $W(t)$ to be non-negative. An individual can either be tense at various levels $(W>0)$ or completely relaxed (at $W=0$ ). As we will assume that there are outbursts for $W(t) \geq \bar{W}$ (see the sect. 3.1 below for a discussion), the range within which $W(t)$ finds itself in is $[0, \bar{W}[$. Being completely relaxed is therefore the desirable state in this model.

One could associate absence of tension also as complete apathy or depression as opposed to being deeply relaxed. ${ }^{28}$ Such a distinction could be modeled if a second dimension measuring how positive or negative a feeling is were introduced. This would then follow the valencearousal tradition in emotion research (Russell and Barrett, 1999). ${ }^{29}$ Here, stress is seen as something inherently negative. While this does not fully capture all aspects of stress, ${ }^{30}$ this analysis focuses on the negative aspects of stress.

The literature on the highly pleasurable state of being "in flow" (e.g. Nakamura and Csikszentmihalyi, 2001) states that demand $p(t)$ can be too high but also too low. When $p(t) / a(t)$ is just at the right level, the individual is "in flow". When demands go up, the individual "first becomes vigilant and then anxious", i.e. a mismatch in the Lazarus sense arises and stress builds up. If demand falls below the level that is just right, an individual "first relaxes and

\footnotetext{
${ }^{28}$ In fact, Selye, who borrowed the term "stress" from physics and introduced it into medical and social sciences argues "complete freedom from stress is death" (Selye, 1976, p. 137). He also developed the distinction between positive stress (eustress) and negative stress (distress). In this language, the present paper is on distress.

${ }^{29}$ See Fontaine et al. (2007) for an argument that an additional two dimensions (potency-control and unpredictability) need to be taken into account. Lerner and Keltner (2000) argue that two emotions with the same valence can imply very different perceptions e.g. of risk.

${ }^{30}$ For an analysis of potential positive effects of stress via subsequent "growth processes" of an individual, see e.g. Karlsen et al. (2006) and the references therein.
} 
then becomes bored". One could therefore look at our lower limit of $W=0$ as the state of "in flow" and analyse boredom as a negative value for $W$ in future work.

\section{Optimal coping}

Given the origins of and means to control tension and given the effect of tension on utility as described in the previous section, we can now start thinking about how an individual behaves optimally in such a setup.

\subsection{The maximization problem}

Any meaningful maximization problem requires trade-offs. So far, only positive effects of coping activities $m(t)$ were discussed: Coping reduces stress and thereby increases expected utility. Coping measures take time, however. This is true for talking to friends or to professional therapists or for practicing sports. We therefore include utility costs from coping $v(m(\tau))$ into the instantaneous utility function.

Outbursts are also effective in reducing emotional tension. At the same time, however, emotional outbursts are associated with high immediate (e.g. reputation) costs. To model these costs in a parsimonious and consistent way, we derive these costs from forgone time as well. Imagine an emotional outburst is a predetermined path $\bar{m}(\tau)$ of intense shouting, drinking, hitting or becoming violent over a period of length $\Lambda$. The present value of utility costs associated with this path not under the control of the individual can then be defined as

$$
v^{M} \equiv \int_{s}^{s+\Lambda} e^{-\rho[\tau-s]} v(\bar{m}(\tau)) d \tau
$$

This captures the idea that the individual is "overwhelmed" by circumstances such that costs are no longer smoothly chosen. ${ }^{31}$

Given utility from consumption and two sources of costs from coping, we can define our utility function as

$$
U(t)=E_{t} \int_{t}^{\infty} e^{-\rho[\tau-t]}[u(c(\tau), W(\tau))-v(m(\tau))] d \tau-\Sigma_{i=1}^{n} e^{-\rho\left[\tau_{i}-t\right]} v^{M} .
$$

We denote the points in time of outbursts by $\tau_{i}$ and subtract the implied present values from utility from consumption and smooth coping. If there are no outbursts, i.e. if $\tau_{i}=\infty$, there are no costs from outbursts and the sum in the objective function "disappears", i.e. equals zero.

The individual maximizes (6) subject to the constraints (5) and (4) by choosing a path $\{m(\tau)\}_{t}^{\infty}$ of calm coping and, possibly, the instants of outbursts. These instants can be characterized by the points in time $\left\{\tau_{1}, \tau_{2}, \ldots\right\}$ when they occur. When these points in time are uncertain, it is much more convenient to characterize outbursts by threshold levels of state variables (see Stokey, 2008). This means for our case that there is an outburst whenever the tension level hits or exceeds a certain tolerance level denoted by $\bar{W} .^{32}$

\footnotetext{
${ }^{31}$ Card and Dahl (2011) in their study of family violence and football argue that violent behaviour can be the outcome of utility maximizing behaviour or e.g. of arguments that escalate out of control. There are economic models where violence is enjoyed by the perpetrator (see e.g. the unitary family model in Tur-Prats, 2015) or comes at a cost but serves as an instrument (to signal marriage dissatisfaction) as in Bloch and Rao (2002). In both of these papers (and in other violence papers cited therein), violence is a rational choice. Our paper thereby provides an alternative view where violent behaviour is at least partly beyond the control of an individual.

${ }^{32}$ Stress $W(t)$ could exceed the threshold $\bar{W}$ by an initial condition or by a negative surprise. We assume that in both cases, a reduction by $\Delta$ is enough to imply $W(t)<\bar{W}$. An extension allowing for $n$ outbursts such that $W(t)<\bar{W}$ only after a reduction of stress by $n \Delta$ is possible.
} 
This tolerance level can be endogenous, i.e. we can let the individual decide optimally at which level there is an outburst, or we can leave them exogenous. This choice is clearly a matter of which feature of outbursts one would like to emphasize. In the first case, outbursts are modelled as a rational choice of technologies how to control tension. One would add one optimality condition, a "smooth-pasting condition", that makes $\bar{W}$ endogenous. If the discrete technology with large costs but also large gains yields higher returns than the smooth technology with marginal costs but also only marginal gains, there will be an outburst. ${ }^{33}$ One appealing factor of an endogenous $\bar{W}$ is the fact that it would reduce the degrees of freedom of the model. The outburst stress level $\bar{W}$ would be tight to personality factors of the individual which would find some support from personality psychology. If one believes, however, that an individual acts sometimes "beyond one's control", the assumption of an exogenous tolerance $\bar{W}$ seems more appropriate.

From a psychological perspective, some authors define coping mechanisms as voluntary responses (Compas et al. 2001). Skinner and Zimmer-Gembeck (2007) include automatic reactions, which would argue in favour of an exogenous $\bar{W}$, into their concept of coping. If we allow for learning, however, the two concepts can become intertwined. Carver and Connor Smith (2010) write that "distinguishing between voluntary and involuntary responses to stress is not simple; indeed, responses that begin as intentional and effortful may become automatic with repetition". Given that we believe that our main conclusions are independent of the endoor exogeneity of $\bar{W}$ and that an exogenous $\bar{W}$ is more parsimonious, we stick to $\bar{W}$ as being exogenous in our model. ${ }^{34}$ The individual anticipates in his choice of coping $m(\tau)$, however, that outbursts occur at $\bar{W} \cdot{ }^{35}$

\subsection{The optimal amount of coping}

The above problem is a stopping problem with continuous adjustment in between the stopping points $\tau_{i}$ (see e.g. Stokey, 2008). It implies (see app. A.1) a standard first-order condition for calm coping $m(t)$. It weighs the marginal gains from less tension resulting from coping with its marginal utility costs. In addition to this condition, we obtain a new condition specific to the stopping nature of this problem which is the "value matching condition". This condition holds for tension levels at or above the tension level where an outburst takes place, i.e. for $W(t) \geq \bar{W}$. It reads

$$
V(W(t))=V(W(t)-\Delta)-v^{M}
$$

and tells us that the value for all tension levels beyond the tolerance level, $V(W(t))$, must equal the value after the outburst at the reduced tension level, $V(W(t)-\Delta)$, minus the instantaneous discrete utility costs due to the outburst, $v^{M}$. The reduction in tension by $\Delta$ reflects the change in (4) due to an emotional outburst. The Bellman equation plus the firstorder condition and the value matching condition describe optimal behaviour of our individual.

We now need to specify functional forms for functions $F(),. \delta($.$) and G($.$) in (5) and on$ the distributional nature of surprises in (1). Given that there is no formal analysis of stress in psychology or economics, the functional forms suggested here are explorative in nature. This means that we will suggest functional forms that make intuitively sense but this also means at

\footnotetext{
${ }^{33}$ The classic example, whose truth is not fully confirmed (Taubman, 2003), is the shoe banging of Nikita Khrushchev at a 1960 UN meeting in New York when in rage about a speech of a Philippine delegate.

${ }^{34}$ This view is shared with the approach of Benhabib and Bisin (2005) who also take automatic behaviour in their model into account. They let individuals choose, however, whether they want to act in an automatic or in a controlled way.

${ }^{35}$ One could give the individual more control over her environment and let her choose, as one example, hours worked. One can then easily imagine that demand $p(t)$ rises in hours worked. As in standard labour supply models, consumption from (3) would then also increase in hours worked. We discuss the implications of such an extension further below once we have studied our existing setup.
} 
the same time that their structure needs to tested or their parameters need to be estimated in subsequent work. The functional forms read

$$
\begin{aligned}
v(m) & =v_{0} m^{1+\zeta}, \\
F\left(\frac{p}{a}, W\right) & =\phi \frac{p}{a} W, \\
\delta(m, W) & =\delta_{0} W+\delta_{1} m, \\
G(g(t), W(t)) & =-\chi g(t)
\end{aligned}
$$

The costs for smooth coping are described by a standard CES structure. The parameter $v_{0}>0$ will be useful for understanding optimal smooth coping later. The cost function needs to be convex $(\zeta>0)$ for our findings to make sense. This is similar to the many quadratic adjustment cost structures in other papers in economics.

The second functional form combines situational and personal components to describe the source of stress. The parameter $\phi>0$ is a personality parameter which captures the aspects ascribed to the function $F($.$) above in the discussion of (5). Putting two individuals into iden-$ tical circumstances, i.e. exposing them to identical ratios $p / a$, can lead to very heterogeneous building up of stress. An individual with a low $\phi$ will not become as tense as an individual with a high $\phi$. The ratio of pressure relative to ability is proportional to the current tension level $W$. This implies that pressure corrected for ability increases tension the more, the higher tension currently is. This captures the idea that someone already relatively tense will react more "nervously", i.e. will build up tension even more when confronted with additional demands as compared to a person with lower $W$.

The functional form might appear restrictive as we do not allow $p / a$ to be a function of time. One might wish to understand how cyclical pressure (relative to ability) due to more or less demanding days induces stress. Examples include a deadline that is approaching vs. the teaching term that comes to an end or the pressure in recession when a firm is about to shut down relative to an expanding firm in a boom. We leave these highly interesting questions for future work. We will undertake below a comparative static analysis, however, to understand how changes of $p / a$ affect an individual's behaviour.

The third functional form specifies how tension is actually reduced. The first term $\delta_{0} W$ captures the ability of an individual to reduce stress by doing nothing. One can draw an analogy from $\delta_{0}$ to a depreciation parameter e.g. for physical capital. Here, $\delta_{0}>0$ denotes an individual's autonomous stress reduction ability. As time goes by, emotional tension ceteris paribus reduces. The second term captures the intentional coping mechanism of the individual. Coping measures $m$ have a linear effect, given the productivity parameter $\delta_{1}$, on the reduction of tension. The higher $\delta_{1}>0$, the more smooth coping measures reduces stress.

The final functional form fixes the effect of surprises $g(t)$ on tension. We view the random surprise as something objective which translates into a jump of stress via the personality factor $\chi>0$. This factor could be identical to the personality factor $\phi$ that translates daily hassles into building up of stress. Our results do not depend on whether they differ or not. Psychologically speaking, one would have to argue that the appraisal process is the same for daily hassles as for rare events. As this seems questionable in such generality, we allow these two personality factors to differ. We specify surprises to be proportional to the current stress level $W(t)$ for the same reason as we did it with daily hassles: one can expect a very relaxed individual with identical personality $\chi$ to react not as strongly to a surprise $g(t)$ as another individual with a higher tension level. The effect of surprises has a minus sign as a positive surprise reduces the stress level and a negative one increases it.

The functional form for consumption is driven by the cognitive load argument and the functional forms for $c=w l\left([M-M(W)]^{\varepsilon}\right)$ from (3). A simple version sets $\varepsilon=1$ and specifies 
labour supply $l($.$) to be the identity function and M(W)$ to be linear in $W$. This implies that consumption falls linearly in $W$

$$
c=w[M-\kappa W],
$$

where $\kappa>0$ is the marginal effect of stress on pre-emptying the memory system and thereby on reducing consumption. Finally, the functional form for the instantaneous utility function $u(c)$ in $(6)$ reads

$$
u(c, W)=\nu c-\alpha W .
$$

The marginal effect of stress on utility is captured by the parameter $\alpha>0$, marginal utility from consumption is denoted by $v>0$.

For the choices made in (8), a closed-form solution for optimal coping $m$ can be derived under a simple parameter restriction. ${ }^{36}$ We find

Proposition 1 Given the assumptions of functional forms in (8) and a parameter restriction

$$
\rho=\phi \frac{p}{a}-\delta_{0}+(\nu w \mu+\alpha) \frac{\Delta}{v^{M}},
$$

optimal coping chosen by our individual is independent of the current stress level and given by (see app. A.1)

$$
m=\left(\frac{v^{M}}{\Delta} \frac{\delta_{1}}{v_{0}} \frac{1}{1+\zeta}\right)^{1 / \zeta}
$$

The restriction on the parameter space (9) basically requires that, given the choice of parameter values for the right-hand side in (9), the time preference rate needs to be equal to this value and this value needs to be positive. We need to make sure in our comparative static analysis that this condition is always satisfied. We will point out when this restriction will play a role. ${ }^{37}$

Expression (10) for optimal coping reveals a lot of interesting and intuitive properties concerning the choice of emotion regulation. Generally speaking, the determinants of smooth coping measures are the costs $\left(v^{M}, v_{0}\right)$ and benefits $\left(\Delta, \delta_{1}\right)$ from both smooth coping and from outbursts and utility parameter $\zeta$.

Corollary 1 Smooth coping increases in $v^{M}$ and $\delta_{1}$ and falls in $\Delta$ and $v_{0}$. The effect of the concavity of the cost function as measured by $\zeta$ is ambiguous. ${ }^{38}$

The rise in $\delta_{1}$ is clearly a productivity issue. When the tension reduction effect of coping rises, calm coping becomes more attractive and more communication takes place. On the other hand, higher costs $v_{0}$ of coping decreases its intensity. When the cost of outbursts $v^{M}$ increase, calm coping measures also increase. This shows the trade-off between these two means of reducing tension. If the alternative (outbursts) is more costly, if talking is more efficient, the amount of talking rises. By contrast, if productivity of outbursts increases, if $\Delta$ becomes larger, why spend a lot of time and effort with smooth coping? Rather using the "tough technology", rather shouting and swearing occasionally is the preferred approach to becoming calm again.

\footnotetext{
${ }^{36}$ There is a long tradition for closed-form solutions in economics, finance and beyond. Closed-form solutions under parameter restrictions have been used at least since Merton (1975). See Wälde (2005) for an application in the analysis of endogenous growth cycles. A recent survey and extensions for closed-form solutions is in Wälde (2011). See also the references in the introduction to optimal stopping problems for further examples.

${ }^{37}$ Further analysis has shown that that there are closed-form solutions for other functional forms. As is well understood, solutions for almost any functional form but for specific quantitative values of parameters can be obtained by numerical methods.

${ }^{38}$ Marginal cost of coping, $(1+\zeta) v_{0} m^{\zeta}$, unambiguously rises in $\zeta$ as long as $m>1$. When optimal communication levels are smaller than 1 , the effect becomes ambiguous.
} 
Interestingly, coping intensity is independent of the current stress level $W$. Technically speaking, we obtain this result as the value function $V($.$) in our closed form solution (see app. A.1)$ falls linearly in $W(t)$. This means, intuitively speaking, that the reduction in the present value $V$ of life-time utility when behaving optimally is independent of the level of tension. When tension reduces by one unit, life-time utility $V$ always reduces by the same constant fraction. Reducing tension is always good, but it is never better or worse to reduce tension at a high tension level than to reducing it at a low tension level. Clearly other functional forms in (8) would imply non-linearities and one can imagine that the attempt to reduce stress by smooth coping can rise in $W .^{39}$

\section{Stress and coping patterns}

\subsection{Dynamics of stress and coping}

\subsubsection{Stress in the absence of surprises}

In a world without surprises, stress evolves in a deterministic manner. What does this model predict about the choice of coping strategies? Would an individual ever be that stressed such that an outburst would occur? Can outbursts be predicted with certainty in a deterministic world? Or would the individual exclusively employ the smooth coping channel to reduce tension? Both can actually occur as we will see shortly.

In a world without surprises, emotional tension described in (5) evolves in a deterministic fashion. Using the functional forms from (8), tension follows (see web app. B.2.2)

$$
\dot{W}(t)=\Phi W(t)-\delta_{1} m
$$

where $\dot{W}(t) \equiv d W(t) / d t$ denotes the time derivative. This equation employs the definition of $\Phi$ as the 'growth rate of stress', a quantity which is central for all the results to follow,

$$
\Phi \equiv \phi \frac{p}{a}-\delta_{0}
$$

Smooth coping intensity $m$ in (11) is now the optimally chosen level from (10). Whenever the tension level $\bar{W}$ is hit, an outburst occurs and tension reduces by a discrete amount as described in (4), $W=\bar{W}-\Delta$.

To understand (11) and (12), let us view pressure relative to ability as something objective, something that can be measured in a fairly consistent way. As an example, think of the number of students asking for advice per day relative to your stock of experience in advice giving or in handling students, measured e.g. in number of student contacts since tenure. How this relative pressure then affects tension building up depends a lot on individual personality as captured by $\phi$. Individuals with a high $\phi$ experience a faster increase in emotional tension, given a certain relative pressure, than others. The growth rate of stress is also characterized by the individual's autonomous stress reduction mechanism, captured by $\delta_{0}$. For individuals with a high $\delta_{0}$, taking a deep breath is enough to be "back to zero", i.e. to quickly digest anything that might have increased tension. For others with a low $\delta_{0}$, it takes a very long time to reduce tension. Finally, there are smooth coping measures allowing to reduce tension. The term $\delta_{1} m$ captures the flow

\footnotetext{
${ }^{39}$ Returning to the issue of an endogenous labour supply discussed in footnote 35 , we would obtain an additional first-order condition fixing hours worked. We would conjecture that labour supply falls in the current stress level and, for a given stress-level, is higher for stress-resistant individuals than for stress-prone individuals (see the definitions below). Stress-prone individuals might actually quit their job completely by setting labour supply to zero. A precise analysis requires a numerical solution of the Bellman equation and is therefore left for future research.
} 
of stress-reducing effects of coping $m$ given a productivity parameter $\delta_{1}$. Coping stands e.g. for the number of hours per day talking to friends. Individuals with high productivity $\delta_{1}$ talk about an issue for a couple of minutes and work out for themselves the essential points while for others it takes hours and a repeated coming back to the same points over and over again to obtain the same reduction in tension.

- Properties of the differential equation for stress

We start by setting $\dot{W}(t)=0$ and find a steady state stress level at

$$
W^{*}=\frac{\delta_{1} m}{\Phi} .
$$

When the stress level of an individual is at this level, it does not change. The question arises, however, whether this stress level is positive or negative and, more importantly, whether it is stable or not. If it is unstable, any small disturbance of the equilibrium tension level would push the individual on a trajectory where the stress level changes over time. A long-run stationary equilibrium might actually not exist and tension could permanently change.

The dynamic predictions of the model are pinned down once we fix the sign of $\Phi$ in (11) and the level of $W^{*}$ in (13). Figure 2 illustrates two of the three qualitatively relevant cases and thereby allows to understand the dynamics of stress and coping.

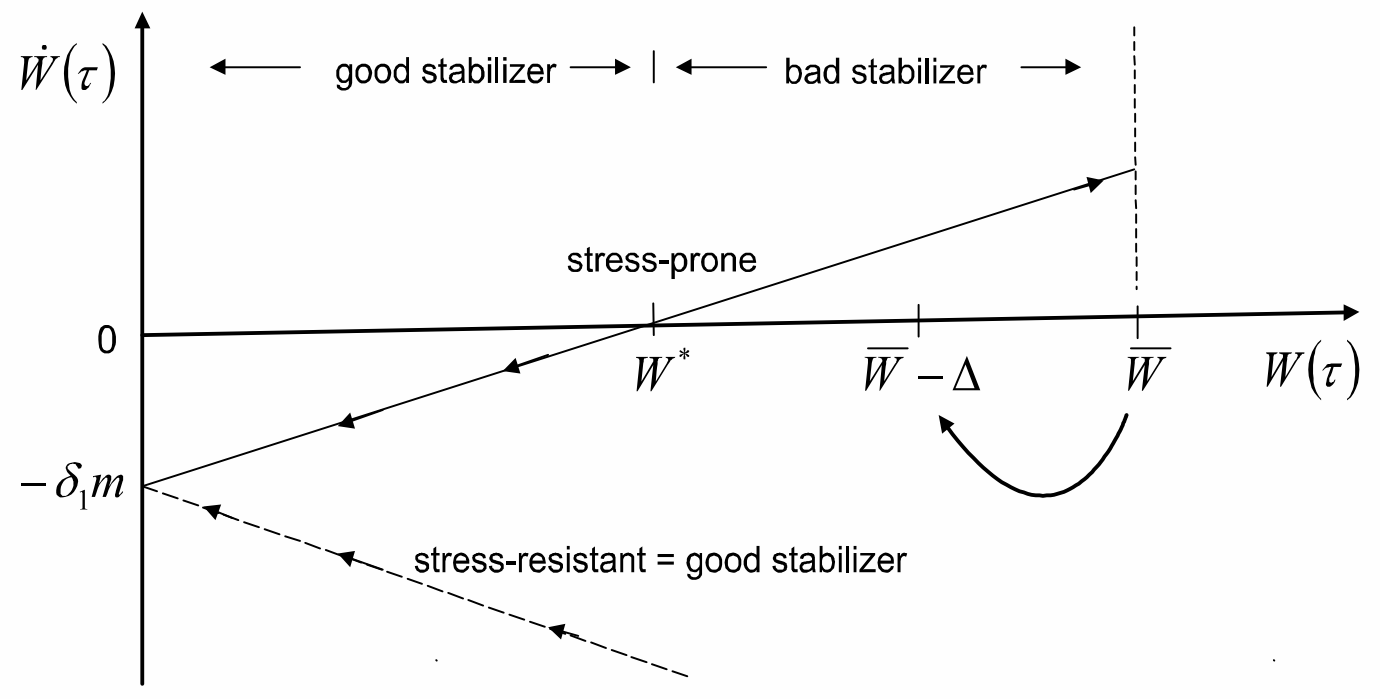

Figure 2 The phase diagram for the dynamics of stress $W(t)$

The horizontal axis plots the tension level $W(t)$ while the vertical axis shows its change over time. For a tension level of zero, the change in tension from (11) is given by $\dot{W}(t)=-\delta_{1} m$. Stress would (technically) reduce further at $W=0$. As stress can not become negative by normalization, the point $\left(0,-\delta_{1} m\right)$ is a stable steady state. Any small disturbance to emotional tension would always bring the individual back to this steady state. As discussed after (5), this can be associated with being "in flow".

As $-\delta_{1} m<0$, an assumption on the slope $\Phi$ of $\dot{W}$ in (11) fixes the sign of the steady state $W^{*}$ from (13). To understand different patterns of stress and coping, the figures distinguishes between a positive and a negative slope $\Phi$. When the slope is positive, there is a second (positive) steady state at $W^{*}$. When the slope is negative, the sign of the corresponding steady state would be negative. As fig. 2 unambiguously shows, the steady state at $W^{*}$ is unstable. Stress falls to the left of $W^{*}$ but rises to the right. ${ }^{40}$

\footnotetext{
${ }^{40}$ The third qualitatively relevant case occurs when the growth rate of stress is positive but the steady state $W^{*}$ lies to the right of the tolerance level $\bar{W}$. We will return to this case further below.
} 


\subsubsection{The role of surprises}

Surprises make the life of individuals uncertain. Stress levels no longer evolve over time in some deterministically predictable way but are subject to random shocks. The evolution of stress is then described by a generalization to the ordinary differential equation (11). The generalization is captured by the stochastic differential equation

$$
d W(t)=\left\{\Phi W(t)-\delta_{1} m\right\} d t-\chi[h(t)-\mu] d q(t) .
$$

The deterministic part of this stochastic differential equation is identical to (11). The frequency of random shocks is determined by the Poisson process $q(t)$ known from (5). The change in the level of stress following a surprise depends on the surprises per se, as specified in (1) and the individual's evaluation as in (14) specified by (8d). Returning to the evidence reported at the end of sect. 2.1, a positive cash transfer would imply $h(t)>\mu$ and a job-loss or bad weather conditions would correspond to $h(t)<\mu$.

As an implication, an individual faces an uncertain future described by a distribution of stress. Stress will be experienced at various levels within the range $[0, \bar{W}[$ as in the deterministic world. Any negative surprise pushing stress above $\bar{W}$ yields outbursts (or cascades of outbursts) such that $W(t) \in[0, \bar{W}[$ at each moment in time.

\subsection{Theory-consistent personality types}

The predictability of outbursts and the stability or instability of stress can best be understood when introducing the concepts of stress-prone and stress-resistant individuals and of good and bad stabilizers. The idea behind these four definitions is to distinguish between individuals per se (stress-prone or resistant) and between individuals conditional on a given stress level.

Definition 1 (Stress-prone and stress resistant individuals, good and bad stabilizers). An individual that $\left\{\begin{array}{c}\text { occasionally } \\ \text { never }\end{array}\right\}$ displays outbursts is called $\left\{\begin{array}{l}\text { stress-prone } \\ \text { stress-resistant }\end{array}\right\}$. An individual whose stress level $\left\{\begin{array}{l}\text { is } \\ \text { is not }\end{array}\right\}$ converging to a level implying an outburst is called a $\left\{\begin{array}{l}\text { bad } \\ \text { good }\end{array}\right\}$ stabilizer.

This definition is most informative for a world without surprises. It is obvious that with sufficiently frequent or strong negative surprises, every individual can display outbursts. It is also informative under the natural assumption that we consider individuals only whose initial stress level lies within $[0, \bar{W}[$. With an exogenous initial stress level higher than $\bar{W}$, an outburst would also be observed for any individual.

- The stress-prone individual

A stress-prone individual is characterized in fig. 2 by the line going through the steady state $W^{*}$. If the current tension level is only a tiny amount below $W^{*}$, the stress level falls over time. The steady state $W^{*}$ can therefore rather be called a threshold level above which the stress level rises. The latter feature is actually the idea behind the labeling "stress-prone". A stress-prone individual is characterized by personal abilities $a$, personality characteristics $\phi$ and stress processing abilities $\delta_{0}$ such that for the given level of demands $p$, the future evolution of tension crucially depends on the current tension level. If the individual is relatively calm (the current $W$ is below the threshold $W^{*}$ ), the individual smoothly reduces tension by consciously chosen smooth coping $m$ and by the autonomous process captured by $\delta_{0}$. If this individual 
happens to have a high current stress level with $W$ being above the threshold $W^{*}$ (see below for surprises and how they can push the individual above or below $W^{*}$ ), stress will continue to rise over time! The individual is doomed to hit the maximum stress level $\bar{W}$ where an emotional outburst will occur.

For such an individual one can predict for any tension level whether an outbursts will occur. For $W>W^{*}$, the time span until the next outburst and the frequency of outbursts can be computed (see prop. 4 below). To make a simple example: Imagine a person working in a team and a colleague notorious for occasional outbursts. If the person has the feeling that the colleague's tension level lies above the threshold $W^{*}$, the person should make sure not to see the colleague too often during the day. Otherwise the person might be hit by an outburst.

The speed with which an individual's stress level changes is an exponential function of time $\tau$ and described by the solution to (11) that reads (see web app. B.2.2)

$$
W(\tau)=\left(W(t)-W^{*}\right) e^{\Phi[\tau-t]}+W^{*} .
$$

The growth rate of stress $\Phi$ displayed in this equation and defined in (12) has an interesting interpretation: Current requirements $p$ and ability $a$ translate into stress accumulation as a function of personality characteristic $\phi$, measuring how strongly $p / a$ affects the individual psychologically. ${ }^{41}$ At the same time, however, the individual is characterized by an autonomous stress reduction process whose speed is driven by $\delta_{0}$. If the individual "takes things to heart", i.e. has a high $\phi$ and at the same time is not good at digesting stress autonomously, i.e. $\delta_{0}$ is low, stress levels change very quickly.

Note however that the individual's situation $(p / a)$ and personality characteristics $\left(\phi\right.$ and $\left.\delta_{0}\right)$ both describe how quickly a stress prone individual accumulates and reduces stress. Consider the situation where the stress-prone individual is a bad stabilizer, i.e. where the current stress level $W(t)$ in $t$ is to the right of $W^{*}$ in fig. 2. Then $W(t)-W^{*}>0$ in (15) and the stress level is accumulating at the rate of $\Phi$. A stress-prone individual can also be a good stabilizer where the tension level is decreased over time. Here as well, the speed with which this happens is $\Phi$. Hence, the rate $\Phi$ can actually also be considered as a measure of emotional stability of an individual. For a given initial stress level $W(t)$, individuals with lower $\Phi$ (and as long as $\Phi$ is positive) will experience a slower change of their stress level than individuals with higher $\Phi$. In some sense, behaviour of individuals with lower $\Phi$ can more easily be predicted than behaviour of individuals with higher $\Phi$.

- The stress-resistant individual

While stress-prone individuals can be good or bad stabilizers, stress-resistant individuals always experience falling stress levels, independently of their current stress level. Stress-resistant individuals are therefore good stabilizers. Whether an individual is stress-resistant or not entirely depends on the outburst level $\bar{W}$ and the growth rate $\Phi$ of stress from (12). For a given $\bar{W}$, the individual becomes stress-resistant as soon as $\Phi$ is sufficiently small. While a negative $\Phi$ as drawn in fig. 2 is a sufficient condition for being stress-resistant, this would not be necessary. If $\Phi$ only becomes small enough such that stress falls for all stress levels between 0 and $\bar{W}$, the individual is stress resistant. The speed with which tension changes is equally described by (15).

\subsection{The outburst theorem}

Let us now turn to the question under which conditions emotional outbursts will (and will not) take place. The question about outbursts is at the same time the question about the

\footnotetext{
${ }^{41}$ One might even want to argue that $\phi$ is negative for some individuals who just get positively aroused from successfully completing tasks. We restrict our analysis here to positive $\phi$.
} 
optimal choice of stress-reduction technologies. In psychology, Gross (2008) in a survey article on how individuals "try to influence which emotions we have, when we have them, and how we experience and express these emotions" (p. 497) writes that "one intriguing puzzle is why people use one emotion regulation strategy rather than another" (p. 505). Seeing stress as an emotion, our findings on the occurrence of outbursts and the determinants of smooth coping provides some answers.

By construction, emotional outbursts take place when the stress level hits or exceeds the outburst level $\bar{W}$. Apart from bad surprises, this can happen only for bad stabilizers. The question is therefore under which conditions an individual turns, first, into a stress-prone individual and, then, into a bad stabilizer. We characterize an individual by personality $(\phi, a, \bar{W})$, by parameters that affect stress regulation only $\left(v_{0}, \zeta, \delta_{0}, \delta_{1}, v^{M}, \Delta\right)$, by the situation $(p)$ and by the current tension level $W(t)$.

The central relationships are in the following

Proposition 2 (Outburst theorem) An individual is

(i) stress-prone if and only if (a) the growth rate of stress is positive, $\Phi>0$, and (b) the threshold level is smaller than the tolerance level, $W^{*}<\bar{W}$.

(ii) These two conditions are satisfied iff

$$
\phi \frac{p}{a}>\frac{\delta_{1} m}{\bar{W}}+\delta_{0}
$$

(iii) The individual turns into a bad stabilizer when (16) holds and the current stress level $W(t)$ is larger than $W^{*}$. This is the case for

$$
\phi \frac{p}{a}>\frac{\delta_{1} m}{W(t)}+\delta_{0}
$$

Outbursts will occur in finite time.

Proof. See app. A.2

Condition (16) as an equality is depicted in fig. 3 as the thick solid line marked $W^{*}=\bar{W}$. The horizontal axis plots the individual's autonomous stress reduction ability $\delta_{0}$. The vertical axis plots $\phi \frac{p}{a}$ as these parameters occur jointly only. Condition (16) as an equality separates stress-prone individuals above this line from stress-resistant individuals on or below the line. When (16) holds as an inequality, the individual lies above the thick solid line.

Whether a stress-prone individual displays outbursts depends on the current stress level. When we fix the stress level $W(t)$ and ask which type of individual would display outbursts in finite time, then we obtain (17). All individuals with $\phi p / a$ sufficiently high such that (17) holds as an inequality, can cope with such a stress level only by employing the outburst-technology as a means to reduce stress.

When we then ask under which conditions outbursts do not occur, i.e. when we find an individual below the thick solid line, then this can be actually true for two reasons. First, an individual is stress resistant per se, i.e. even when coping measures are absent $(m=0)$. This is the case for a negative growth rate of stress, $\Phi<0$. Or an individual is stress-resistant due to a sufficiently high level of smooth coping measures. These individuals are characterized by a positive growth rate of stress, $\Phi>0$. But this rate is so low, given the negative intercept $-\delta_{1} m$, that the threshold level $W^{*}$ lies to the right of (or just at) $\bar{W}$. Outbursts therefore never occur (in the absence of negative surprises). ${ }^{42}$

\footnotetext{
${ }^{42}$ The case of $W^{*}>\bar{W}$ is the third qualitative case. It was not depicted in fig. 2 which only displays two cases.
} 


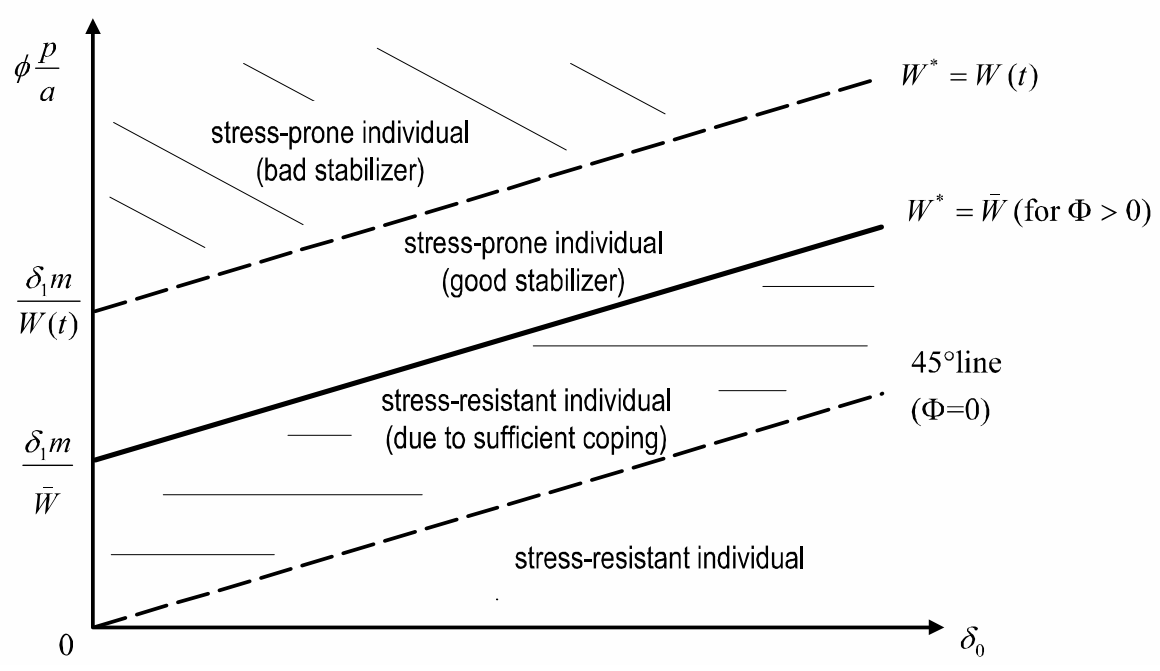

Figure 3 Stress-resistant and stress-prone individuals and regions of bad stabilizers implying emotional outbursts

This figures nicely allows us to summarize conditions for being stress-resistant and stressprone in the following

Corollary 2 (Determinants of emotional outbursts)

(a) For a given autonomous stress-reduction potential $\delta_{0}$, an individual will be (unconditionally) stress-resistant if $\phi p / a$ is sufficiently low.

(b) For a given autonomous stress-reduction potential $\delta_{0}$, an individual will be stress-resistant if coping activities are sufficiently high or the tolerance level $\bar{W}$ is sufficiently low.

(c) For a given autonomous stress-reduction potential $\delta_{0}$, a given coping level $m$ and a given tolerance level $\bar{W}$, an individual will be stress-prone if $\phi p / a$ is too high.

(d) An individual is a bad stabilizer if $\phi p / a$ rises further.

We see from the above findings that, taking a situation $p$ and an individual's ability $a$ as fixed, personality is crucial. The appraisal parameter $\phi$ and stress reduction abilities $\delta_{0}$ determine whether an individual is stress-resistant or stress-prone. How one looks at the world, i.e. how an individual perceives and evaluates stressors is crucial for determining whether the individual will display emotional outbursts or not. If $\phi$ is only sufficiently small (however large the intensity of stressors), the individual will remain calm and not display outbursts. Similarly for the autonomous stress reduction ability $\delta_{0}$, when we fix $\phi p / a$ and move horizontally through the figure. If it is only sufficiently high, an individual will not display outbursts.

What is the role of controlled coping $m$ ? For a given set of parameters (other than those fixing $m$ ), any parameter that increases $m$ reduces the risk of an outburst. Trying to reduce one's tension level e.g. by talking through stressors and their implications for one's well-being should help. One can even say more: For a given set of parameters and a given tension level $W(t)$, there is a coping intensity $m$ that makes sure that outbursts can be avoided.

\subsection{Temporary stressors and permanent stress?}

Let us now return to our world with uncertainty, i.e. we now allow for positive and negative surprises as presented in (1). The impact of surprises can also be best understood when we distinguish between stress-resistant and stress-prone individuals. Fig. 4 shows the evolution of stress for a given realization of surprises. 
We assume that both individuals are good stabilizers to start with. As a consequence, their tension levels fall. Now we allow for a negative surprise after a day, i.e. at $t=24$, meaning that $h(t)-\mu<0$. As a consequence, stress of both goes up by the same amount given by $d W(t)=\chi[h(t)-\mu]$ from (14) with $d q(t)=1$ and $d t=0$ (meaning the jump is instantaneous). We assume that the increase in stress is not enough to push the stress-prone individual above the threshold level $W^{*}$. Both individuals therefore continue reducing their stress level.

When the next surprise comes early (the stress level is still high) or the negative surprise is stronger, stress levels of both individuals will be pushed up to an even higher level than before. This takes place at $t=38$ in our figure. The subsequent evolution of stress for the stress-prone and the stress-resistant individual are now different. The stress-prone individual will now experience a rise in stress while the stress level of the stress-resistant individual goes down. An identical history of surprises leads to an almost identical history of stress up to the point where stress crosses the threshold level $W^{*}$ for the stress-prone individual. As of this level, the stress-prone individual is a bad stabilizers and is heading towards an emotional outburst. The outburst takes place in this example at $t=91$. The stress-resistant individual's stress level keeps falling over time.

Evolution of stress under surprises

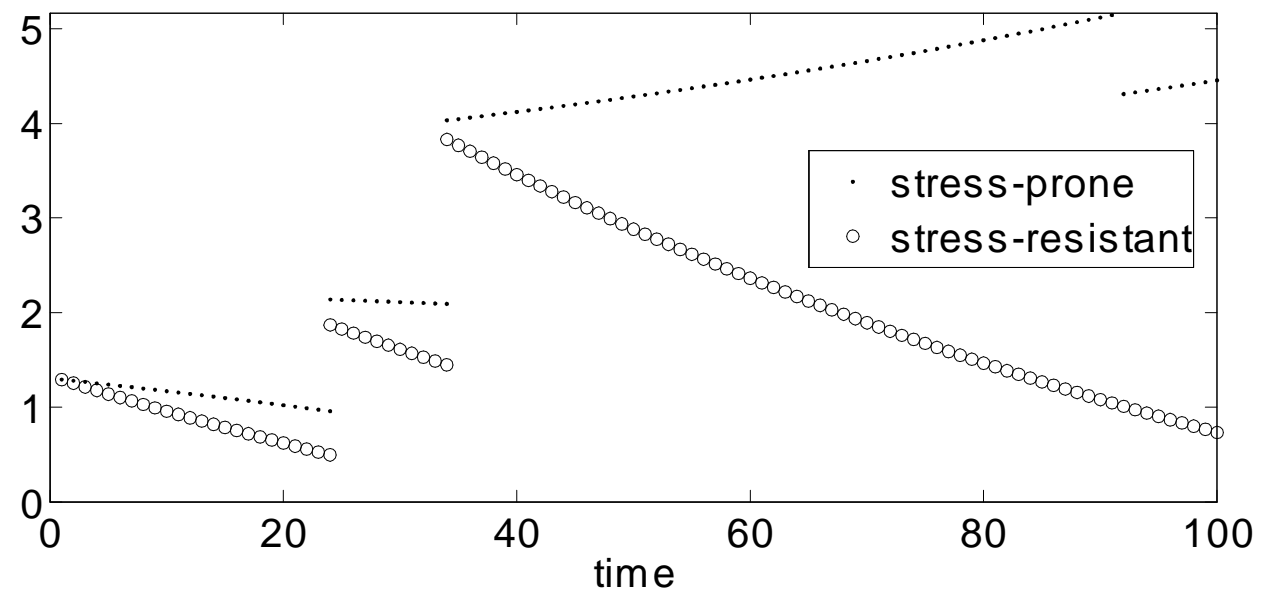

Figure 4 Emotional tension for a stress-resistant a stress-prone individual (right panel) under identical surprises

While fig. 4 just illustrates one possible realization for surprises and the evolution of stress for stress-resistant and stress-prone individuals, this example points to a more general finding.

Proposition 3 A temporary surprise only temporarily changes the stress level of an individual if the individual is stress resistant. A temporary surprise will have a permanent effect on an individuals stress level if (i) the individual is stress prone and (ii) if the temporary surprise pushes the individual's stress level above or below the threshold level $W^{*}$.

Clearly, a temporary surprise for a stress prone individual can make changes towards the good or the bad. A positive surprise can push the individual from outbursts that occur at the frequency (18) to an evolution of stress that eventually leads to being fully relaxed. In the bad case, a negative surprise would lift the individual above $W^{*}$ and frequently recurring outbursts would result.

This finding helps to inform further empirical work e.g. on the effect of poverty alleviation on psychological well-being. Haushofer and Fehr (2014) conclude that "little is known about whether poverty alleviation leads to a permanent or only temporary increase in psychological well-being". Our theoretical analysis suggests that it depends on (i) how strong a positive surprise (through poverty alleviation) is and on (ii) the personality of a person. 


\section{$5 \quad$ How to deal with emotional outbursts?}

We have now understood under which conditions emotional outbursts occur. We now take a slightly enlarged perspective and ask what the effects were if an individual could change the tolerance level. We also ask how often outbursts can occur and how an individual displaying outbursts could react to them in some deeper (personality) sense.

\subsection{Is suppressing outbursts a good idea?}

Given the general belief that emotional outbursts are not desirable from a social point of view, a bad stabilizer might feel encouraged to increase the tolerance level $\bar{W}$. Imagine an individual could train self-control to inhibit or at least postpone the outburst to a higher stress level. What is the effect of a higher $\bar{W}$ ?

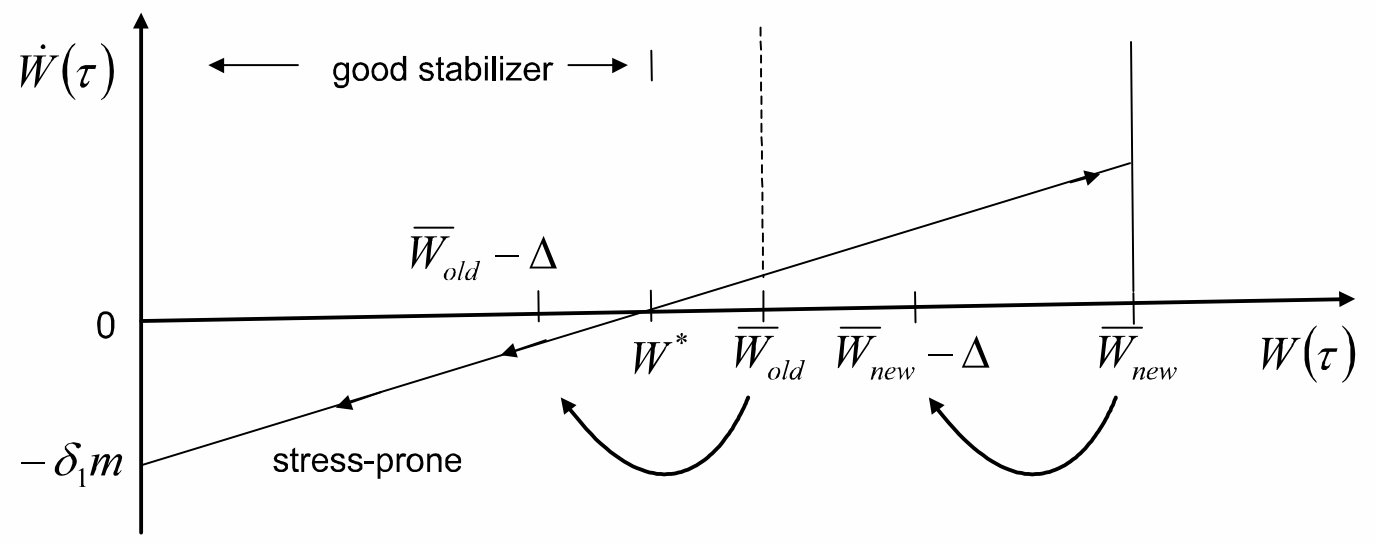

Figure 5 The effect of a higher outburst level $\bar{W}$

We start by looking at a stress-prone individual. One effect of a higher $\bar{W}$ is definitely desirable - the next outburst comes later. Starting an epsilon to the right of the threshold level $W^{*}$, the outburst occurs later when the outburst level is $\bar{W}_{\text {new }}$ as compared to $\bar{W}_{\text {old }}$. Leaving the outburst level $\bar{W}_{\text {old }}$ where it is, however, implies that an outburst by (4) pushes the stressprone individual to the left of the threshold level $W^{*}$. The individual therefore becomes a good stabilizer, reduces stress and eventually ends up at the stress-free level $W=0$. An outburst truly helped the individual in permanently reducing stress.

If the same individual had an outburst at the higher level $\bar{W}_{\text {new }}$, the outburst would also reduce stress by the discrete amount of $\Delta$. Now, however, the individual remains a bad stabilizer. The individual experiences rising stress levels again and will soon have the next outburst. With a higher $\bar{W}_{\text {new }}$, the individual is caught in an outburst cycle: When at $\bar{W}_{\text {new }}-\Delta$, stress increases smoothly and only controlled coping behaviour is observed. Once at $\bar{W}_{\text {new }}$, an outburst takes place and the individual is thrown back to $\bar{W}_{\text {new }}-\Delta$ and so on. Increasing the outburst level therefore might lead to more rather than to fewer outbursts.

\subsection{The frequency of outbursts}

Having understood conditions for the occurrence of outbursts, we now ask how frequently they occur. If an individual is very good at reducing the tension level via an outburst, i.e. if $\Delta$ is sufficiently large, an outburst will happen only once and never again. This is the situation described in fig. 5 for $\bar{W}_{\text {old }}$. If the individual is not that talented and an outburst predicts the next outburst (as with $\bar{W}_{\text {new }}$ ), one can compute the frequency with which outbursts occur. This is summarized in the following 
Proposition 4 (Frequency of emotional outbursts)

(i) A bad stabilizer with a low effectiveness $\Delta$ of outbursts will experience outbursts with a frequency of $T^{-1}$ per unit of time where

$$
T^{-1}=\Phi / \ln \frac{\bar{W}-W^{*}}{\bar{W}-\Delta-W^{*}} .
$$

(ii) The frequency $T^{-1}$ of outbursts rises, the higher the growth rate of stress $\Phi$, the higher the outburst level $\bar{W}$ and the lower the threshold level $W^{*}$,

$$
\frac{d T^{-1}}{d \Phi}>0, \frac{d T^{-1}}{d \bar{W}}>0 \text { and } \frac{d T^{-1}}{d W^{*}}<0
$$

(iii) The effect of $\Delta$ on the frequency $T^{-1}$ of outbursts is ambiguous.

Proof. see app. A.3

The result that the growth rate of stress $\Phi$ increases the frequency of outbursts is due to the finding discussed after (15) that a high growth rate of stress leads to fast changes of stress level. A bad stabilizer experiencing an outburst leading to a discrete and fast reduction of stress will see stress build up quickly again, which then leads to the next outburst.

The result that a higher outburst level leads to a higher frequency of outbursts again indicates that outbursts at high stress levels might not be as desirable as outbursts at low stress levels. This reminds of the finding discussed in fig. 5. Here the higher frequency of outbursts at higher stress levels result from the fact that speed with which stress grows is faster, the further the current stress level is distant from the threshold level $W^{*}$. Looking at (15) clearly shows that if the initial stress level $W(t)$ after an outburst, i.e. $\bar{W}-\Delta$ is higher, the speed of stress accumulation - at an invariant growth rate of stress - rises.

The finding on the steady state level $W^{*}$ is comforting. The steady state rises, inter alia, in optimally chosen coping measures $m$. The more an individual finds it optimal to e.g. talk to a friend, the lower the frequency of outbursts. The reason for this lies again in the speed of stress accumulation at an invariant growth rate of stress. When $W^{*}$ is high, the stress level $\bar{W}-\Delta$ after an outburst can not be too far from $W^{*}$ such that it takes a lot of time for accumulating stress again.

The ambiguous effect of the efficiency $\Delta$ of an outburst results from two channels. The direct effect of $\Delta$ decreases the frequency as can be seen from (18). The efficiency $\Delta$ also has an indirect effect via optimal coping $m$ and thereby via the threshold level $W^{*}$. A higher outburst efficiency makes outbursts more attractive as compared to smooth coping (10). Less coping reduces the threshold level and therefore increases the speed with which stress is accumulated (the time derivative $\dot{W}$ to be precise) at $\bar{W}-\Delta$ and higher. The frequency of outbursts therefore increases through the indirect channel. The overall effect is therefore ambiguous.

\subsection{The gains from psychotherapy}

Imagine our individual could undergo psychotherapy. This would allow him to influence some appraisal processes or undertake some reappraisals, i.e. adjust the way he evaluates certain stressors. In terms of our model, this would mean that the individual would be able to adjust certain personality parameters like $\phi, \delta_{0}$ and $\delta_{1}, \chi$ and $\mu$ or even opt for a different environment that provides a lower intensity $p / a$ of stressors (e.g. change jobs). The former would be examples of an emotion-focused strategy and the latter of a problem-focused strategy to reduce stress. Even though endogenizing the costs of going through therapy has to be postponed to future research, we can still provide measures of the benefits of such therapy. ${ }^{43}$

\footnotetext{
${ }^{43}$ I am grateful to Ernst Fehr for having suggested that such an analysis can be undertaken in this context.
} 
If we want to understand how emotion- or problem-focused therapies would allow to reduce the occurrence of frequency of outbursts, we can simply go back to the outburst theorem in prop. 2 or to the frequency results in prop. 4 . We would then find that outbursts can be avoided if the following changes are sufficiently large: (i) coping measures $m$ increase, (ii) the intensity of stress is appraised in a less threatening way, i.e. if $\phi$ goes down, (iii) the autonomous stress reduction ability $\delta_{0}$ rises or (iv) the productivity $\delta_{1}$ with which coping measures reduce tension increases.

At some deeper level, however, one would like to understand how therapy affects wellbeing of an individual and not just a certain characteristic behaviour. Empirically speaking, measures of well-being can be obtained in at least in two ways: subjective reporting based on questionnaires or on experience sampling (see e.g. Krueger and Schkade, 2008). Answers to questionnaires are usually understood to be a highly cognitive, effortful and reflective process. By contrast, a spontaneous report of a current feeling by experience sampling at repeated but random points in time seems a more immediate and less cognitively controlled measure. To the extent that the value function is a measure that takes current state variables and the entire future of control variables into account, the value function sounds more like the outcome of a reflective process. We therefore feel justified in equating the value function to subjective well-being and make a prediction about the effects of psychotherapy on subjective well-being as reported in questionnaires. ${ }^{44}$ We start from the following

Proposition 5 (Subjective well-being) Let the parameter restriction (9) hold, i.e. $\rho=\phi \frac{p}{a}-$ $\delta_{0}+(\nu w \kappa+\alpha) \frac{\Delta}{v^{M}}$. Subjective well-being, aka the value function $V(W)$ of the individual, is then given by

$$
V(W)=\Lambda_{0}-\frac{v^{M}}{\Delta} W
$$

where

$$
\rho \Lambda_{0}=\nu w M-v_{0} m^{1+\zeta}+\frac{v^{M}}{\Delta}\left[\delta_{1} m+\lambda \chi\left[E^{h} h-\mu\right]\right]
$$

and optimal coping $m$ is given by (10), i.e. by $m=\left(\frac{v^{M}}{\Delta} \frac{\delta_{1}}{v_{0}} \frac{1}{1+\zeta}\right)^{1 / \zeta}$.

\section{Proof. See app. A.1}

The value function has intuitive properties. First, it falls in the stress-level $W$, where the slope is given by the ratio of the cost $v^{M}$ to the benefit $\Delta$ of the outburst. Second, the value for a fully relaxed individual $(W=0)$ is given by $\Lambda_{0}$ which captures many aspects coming from the structure of the Bellman equation. They include on the right hand side of (20) (parts of the expressions for) the benefits from consumption, the costs of coping, the benefits of coping and the influence of surprises.

Given these findings, we can now ask how strongly well-being changes as a result of therapy. Formally, we would like to understand the derivative of the value function with respect to any of the parameters of interest $\left(\phi, \delta_{0}\right.$ and $\delta_{1}, \chi$ and $\mu$ ). This is technically straightforward for $\delta_{1}, \chi$ and $\mu$ : An individual that becomes more productive in using his time for coping measures (just talk 10 minutes to a friend instead of 20 to experience the same amount of stress reduction) enjoys a higher $V(W)$. The result for a decrease of $\chi$ is ambiguous: A priori, a less emotional response to surprises via a lower $\chi$ appears desirable. Equation (20), however, tells us that becoming less emotional can have positive or negative effects. If an individual is on average positively surprised, i.e. if $E^{h} h>\mu$, subjective well-being of the individual actually falls if the person becomes less emotional. Therapy attempting to make an individual emotionally

\footnotetext{
${ }^{44} \mathrm{An}$ immediate implication of this argument states that the appropriate theoretical counterpart for experience sampling would be the instantaneous utility function.
} 
more stable is therefore only advisable if the individual tends to be negatively surprised, i.e. if $E^{h} h<\mu$. If therapy could influence both the degree $\mu$ of optimism and appraisal $\chi$ of surprises, one should aim at reducing $\mu$ and increasing $\chi$. The individual should expect less and become more emotional.

When we want to compute derivatives with respect to $\phi$ and $\delta_{0}$, it is not so clear how such a derivative has to be computed given the parameter restriction (9). For the derivative to be true, we need to make sure that this restriction also holds "after the derivative", i.e. when the parameter is a tiny bit larger. To make sure that this holds, we need to compute derivatives such that the parameter restriction (9) is valid for all values of the parameter we are interested in.

To make an example, we start with $\phi$, the appraisal of daily hassles as captured by the intensity $p / a$ of the stressor. Imagine the individual lives in an environment where daily hassles exceed daily uplifts such that $p>0$. Then, a decrease of $\phi$, i.e. a more "relaxed appraisal" or a "less worried" evaluation of stressors, would by (11) and (12) imply that stress does not increase that quickly. What are the utility effects for the individual? As we cannot compute the derivative of $V(W)$ with respect to $\phi$ given that $\phi$ appears in the constraint (9), we undertake the following thought experiment.

Imagine we want to understand the effect of therapy on an individual that experiences a rise in the intensity of stressors. In other words, we want to understand the effect of a decrease in $\phi$ when $p / a$ increases such that $\phi p / a$ in (9) remains constant. Given this constant $\phi p / a$, we know that (9) holds for any combination of values of $\phi$ and $p / a$ we look at. We can then conclude that therapy allowing an individual to better cope with more stressors is beneficial for the individual: The individual feels just as well (the value function $V(W)$ does not change as the path of stress $W$ is unchanged), even though the intensity of stressors increased.

We can then understand the effect of an increase in the autonomous stress-reduction ability $\delta_{0}$ in the same way. Imagine again the intensity $p / a$ of the stressors goes up. If at the same time the ability $\delta_{0}$ increases such that $\rho$ in (9) remains constant, we can conclude that a therapy increasing $\delta_{0}$ allows the individual to better cope with more stress. (Note that in contrast to the change in $\phi$, where the impact on the stress level was unchanged, here we do get more stress as $\phi p / a$ goes up but this higher propensity of stress creation is immediately compensated for by higher $\delta_{0}$.)

With these lines of reasoning, we can summarize the effects of therapy in the following

Corollary 3 A therapy increases subjective well-being $V(W)$ of the individual if

(i) the individual's productivity $\delta_{1}$ in coping $m$ rises

(ii) the individual becomes emotionally more stable ( $\chi$ falls) conditional on the individual being on average negatively surprised

(iii) the individual becomes more emotional ( $\chi$ rises) conditional on the individual being on average positively surprised,

(iv) the individual reduces expectations with respect to surprises ( $\mu$ falls) as this leads to positive surprises more often.

The results from our thought experiments are summarized in the following

Corollary 4 A therapy allows an individual to keep its level of subjective well-being despite a rise in the intensity of stressors if

(i) the individual succeeds in reacting less emotional (lower $\phi$ ) to daily hassles

(ii) the individual manages to improve his autonomous stress reduction potential (increase $\delta_{0}$ ).

A more general insight from these analyses points to the fact that there is an optimal degree of emotional responses. Just as individuals can be too emotional (when living in worlds with 
negative surprises and daily hassles), individuals might be too little emotional (when living a world with lots of positive surprises and daily uplifts). The optimal outcome would therefore consist in making an individual more emotional (increase $\chi$ ) but let him expect less (decrease $\mu)$. A reduction in $\phi$ making the individual less susceptible to daily hassles and an increase in stress reduction productivities $\delta_{0}$ and $\delta_{1}$ then completes optimization of emotion regulation. A full "theory of therapy" would have to wait for convincing specifications of costs arising from these changes in personality.

\subsection{Structurally estimating personality}

Our setup stresses that there is a large heterogeneity in how stressors translate into stress. Individuals exhibit heterogeneous behaviour in identical situations given their heterogeneous appraisal of stressors. This stressor-stress link is captured by personality traits and coping dispositions in our setup. Looking at (14) with (12), the central parameters in this respect are $\phi$ for translating daily hassle into changes of stress, $\delta_{0}$ and $\delta_{1}$ for how quickly an individual can reduce stress and $\chi$ how strongly an individual reacts to surprises. The general optimism parameter $\mu$ also captures a personality dimension. With this framework, we defined theoryconsistent personality types in sect. 4.2.

It is then a natural question to ask how these parameters and personality types can be reconciled with the more standard measures of personality like e.g. the Big $5 .{ }^{45}$ These five measures are usually called 'neuroticism' ("the ease and frequency with which a person becomes upset and distressed"), 'agreeableness' (agreeable individuals "get less angry over others' transgressions than do less agreeable people") and 'conscientiousness' (in the sense of taking "future contingencies into account", all quotes from Carver and Connor-Smith, 2010). The remaining two personality factors, 'extraversion' and 'openness to experience', play a less important role in our framework.

To systematically establish a link between the personality parameters in the model $\left(\phi, \delta_{0}\right.$ and $\delta_{1}, \chi$ and $\mu$ ) and the questions underlying the Big 5 personality measures, one could proceed as follows. The dependent variable could be behaviour, say the frequency of outbursts, or self-reports on stress $W(t)$. Explanatory variables are stressors $(p$ and $h(t))$ in addition to various control variables (like education, occupation, age etc) that capture inter alia ability $a$. In an exploratory setup, one would estimate the effects of BIG 5 measures on the dependent variable in a linear regression. For a structural interpretation of personality characteristics, however, it would be more interesting to jointly estimate standard model parameters (like preference parameters) with the model's personality parameters. The current state of the art (see e.g. Heckman et al., 2006 or Conti et al., 2014) uses linear simultaneous equation models with latent factors as explanatory variables (see Piatek, 2010 for an introduction). The ideal approach building on models like the one developed here would use the density provided by the theoretical model (e.g. on outbursts or stress) for the formulation of a likelihood function combined with the densities of the latent variables. The difference to existing analyses would consist in the closer link of the personality estimates to the theoretical model resulting from the use of the model density for estimation. This would not only be interesting for the present stress-coping model but also more generally for improving our theoretical understanding of the factors explaining the answers in standard questionnaires for personality.

More in detail, estimation would employ two equations. The first one results from the theoretical model and provides us with a closed-form expression of the density of stress $g\left(W \mid \Theta_{i}, X_{i}, \sigma\right)$. This density is conditional on the latent personality factors $\Theta_{i}=\left\{\phi, \delta_{0}, \delta_{1}, \chi, \mu\right\}$, covariates $X_{i}$

\footnotetext{
${ }^{45}$ See e.g. John et al. (2008) for a psychological perspective and Borghans et al. (2008) for first steps towards integrating economics and personality psychology. Borghans et al. provide, inter alia, a criticism of the atheoretical nature of the Big 5 personality measures and compare it to other taxonomies of personality.
} 
and model parameters $\sigma$ (other than latent personality factors, i.e. $\sigma=\left\{\rho, v_{0}, \zeta, \kappa, v, \alpha\right\}$ )

$$
\begin{gathered}
W_{i}^{\sim} g\left(W \mid \Theta_{i}, X_{i}, \sigma\right), \\
Y_{i}^{*}=X_{i} \beta+\alpha \Theta_{i}+\varepsilon_{i} .
\end{gathered}
$$

The second equation explains latent outcomes $Y_{i}^{*}$ of personality by covariates $X$ (like $p, a$ and $w$ ), latent personality factors $\Theta_{i}$, factor loadings $\alpha$ and the error term $\varepsilon_{i}$. Employing the density from the theoretical model in the likelihood function then makes sure that the estimated personality factors $\hat{\Theta}_{i}$ obtain a very precise meaning. ${ }^{46}$ This would be an advantage not only for the present analysis but also for studies of personality in economics and in psychology more generally.

\section{Conclusion}

The feeling of being stressed is known by everybody. At times, demands on the job, by family or from friends just exceed personal abilities. Demands beyond abilities imply stress. But why is it that some individuals remain calm even in times of highest work load while others start becoming nervous and might even display strong emotional reactions? This paper has presented a model that builds on concepts borrowed from psychology and translates them into standard economic model building. This yields the huge advantage that we can now, to return to Rabin (2013a) again, understand what concepts in psychology actually mean, to understand where the flaws are and to see their limits that guide us in further advancing our knowledge.

We have seen that stress is an inherently dynamic concept. Stress builds up gradually as a function of appraisals of stressors. Stressors can come as a surprise or they are present at each instant. Surprises can be positive or negative, daily stressors can be uplifts, which would reduce stress - again only gradually and slowly - over time. We have also seen how some of the most popular concepts in psychology, cognitive load and mental resource constraints, can easily be introduced into economic models. We have understood how such a resource constraint can be used to easily understand how stress reduces performance of an individual on the job.

When it comes to optimal reactions to stress, this paper formalizes many of the psychological views about functional vs. dysfunctional, problem-focused vs. emotion-focused and reflective (rational) vs. automatic (impulsive) strategies. We understood - using an optimal stopping framework for optimal stress reactions - that the distinction between reflective and impulsive strategies is not so clear-cut after all: If an individual knows about automatic behaviour, it should anticipate this automatic behaviour. In this sense, the automatic behaviour is automatic in this particular situation (technically speaking where the state variable stress $W(t)$ reaches a certain tolerance level $\bar{W}$ ), but this automatic behaviour is anticipated and thereby at least to some extent under the control of the individual. Boundedly rational behaviour combined with rational behaviour becomes semi-boundedly rational behaviour. This can be seen as an implementation of Rabin's (2013b) suggestion to use neoclassical optimization models to integrate limits to rationality.

Our setup allows us to understand stress and coping patterns given optimal stress regulation of an individual. We saw that emotional outbursts, the socially less desirable coping strategy, occurs at predictable points in time. All individuals employ smooth and constructive stresscoping strategies for some time. These strategies help reducing the current stress level (or at least reduce the speed with which stress builds up). But in certain cases, it might be optimal to allow emotional outbursts to take place. After all, socially desirable or not, emotional outbursts are just one out of two technologies to reduce emotional tension. If various technologies are available to achieve a certain goal (here: stress reduction), it is not surprising to see that

\footnotetext{
${ }^{46}$ I am grateful to Rémi Piatek for discussions of aspects related to latent factor models.
} 
optimizing individuals employ both technologies. We have also seen that suppressing outbursts might lead to outburst cycles which would not have occurred if the individual had allowed the outburst to occur immediately.

The entire analysis builds on the distinction between two types of individuals: stress-prone and stress-resistant individuals. The advantage of this definition of individual personality is its theoretical consistency. Psychological measures of personality (think about the Big 5) are based on questionnaire answers and factor analysis. The paper works out which steps would have to be undertaken in order to structurally estimate theory-consistent personality measures. Using this distinction, we have seen that temporary shocks (e.g. a short stressful event at work) can have permanent effects on the stress-level on an individual if the latter is stress-prone. To put it positively, for a stress-resistant individual, any temporary stressful event will not prevent him to eventually return to low stress-levels once the event is over.

What do we learn generally about how desirable emotions are for individual well-being? Surprisingly, it depends on a persons environment and further personality characteristics. If there were means to change a person's personality (think of psychotherapy), one would not necessarily want emotion-free individuals. Emotions can be of a big advantage for wellbeing: If individuals "expect little from the world" (their subjective expectation $\mu$ is low), they are more often positively surprised than they are negatively surprised. If they than value these positive surprises more strongly (their appraisal parameter $\chi$ ), they are very close to an optimal emotional structure.

The analysis suggests many further questions that should be studied in the future. The reaction of individuals to stress beyond stress regulation should be studied jointly with this setup. As discussed in footnote 35, labour supply reactions would display interesting patterns. The role cognitive load plays could be elaborated further. As an example, one might want to take into account that an individual's ability $a$ to react to demand $p$ is a function of cognitive load as well. Stress does not only reduce effective labour input but also reduces an individuals ability to react to stressors. One can also study the role of stress on consumption and saving behaviour. Do stressed individuals consume more to compensate for being stressed? Going beyond the individual level, one can think about the management of firms, about principalagent problems where a principal optimally chooses the amount of pressure put on an agent in order to achieve optimal output. This can then easily be extended to allow for groups and how the stress-level in a group works as a coordination device that helps to coordinate collective action. Interaction between a small number of individuals at the same hierarchy level, as a team in a firm or in an intimate partnership, where outbursts of one team member exerts a negative externality on the other team member, would then be a natural next step to be

analysed. Finally, the effects of stress on health and health costs at the aggregate level are also worth being considered.

\section{A Appendix}

\section{A.1 The solution to the maximization problem}

The Bellman equation for the case where $W(t)<\bar{W}$ reads

$$
\rho V(W(t))=\max _{m(t)}\left\{\begin{array}{c}
u(c(t))-v(m(t))+V^{\prime}(W(t))\left[F\left(\frac{p(t)}{a(t)}, W(t)\right)-\delta(m(t), W(t))\right] \\
+\lambda\left[E^{h} V(W(t)+G(g(t), W(t)))-V(W(t))\right]
\end{array}\right\} .
$$

The first-order condition for $m(t)$ in (A.1) requires

$$
v^{\prime}(m(t))=-V^{\prime}(W(t)) \frac{\partial}{\partial m(t)} \delta(m(t), W(t)) .
$$


Assuming an interior solution, marginal costs from coping, $v^{\prime}(m(t))$, must equal marginal gains, $-V^{\prime}(W(t)) \frac{\partial}{\partial m(t)} \delta(m(t), W(t))$. As stress $W(t)$ is "a bad" and not a good, $V^{\prime}(W(t))$ is negative and the right-hand side of this first-order condition indeed reflects a positive marginal gain. The first-order condition implicitly makes optimal coping a function of tension, $m(t)=$ $m(W(t))$. For $W(t) \geq \bar{W}$, stress reduces instantaneously by (4) and the value matching condition (7) holds.

For the functional forms from (8), the Bellman equation and the first-order condition read

$$
\begin{aligned}
\rho V(W) & =\max _{m}\left\{u(c, W)-v_{0} m^{1+\zeta}+V^{\prime}(W)\left[\Phi W-\delta_{1} m\right]+\lambda\left[E^{h} V(W-\chi g)-V(W)\right]\right\}, \\
(1+\zeta) v_{0} m^{\zeta} & =-V^{\prime}(W) \delta_{1} .
\end{aligned}
$$

We start with a guess $J(W)$ for the value function $V(W)$ which reads $J(W)=\Lambda_{0}-\Lambda_{1} W$. The guess implies $J^{\prime}(W)=-\Lambda_{1}$ and gives us two free parameters, $\Lambda_{0}$ and $\Lambda_{1}$.

We need to verify that this guess satisfies the value-matching condition, the first-order condition and the Bellman equation. The value-matching condition (7) is satisfied if the first parameter $\Lambda_{1}$ satisfies

$$
\Lambda_{0}-\Lambda_{1} W=\Lambda_{0}-\Lambda_{1}[W-\Delta]-v^{M} \Leftrightarrow v^{M}=\Lambda_{1} \Delta \Leftrightarrow \Lambda_{1}=\frac{v^{M}}{\Delta} .
$$

The first-order condition (A.2) is satisfied if $(1+\zeta) v_{0} m^{\zeta}=\Lambda_{1} \delta_{1}=\delta_{1} \frac{v^{M}}{\Delta}$, where the second equality used (A.3). This fixes an optimal coping level $m$ at

$$
m^{\zeta}=\frac{v^{M}}{\Delta} \frac{\delta_{1}}{(1+\zeta) v_{0}} \Leftrightarrow m=\left(\frac{v^{M}}{\Delta} \frac{\delta_{1}}{v_{0}} \frac{1}{1+\zeta}\right)^{1 / \zeta} .
$$

Finally, the Bellman equation with $u(c, W)$ from (8f) and consumption from (8e) holds if two conditions are fulfilled simultaneously (see the web appendix for more details on the rearrangements). The first is the parameter restriction (9) in the main text. The second pins down $\Lambda_{0}$ from our guess which needs to satisfy

$$
\rho \Lambda_{0}=\nu w M-v_{0} m^{1+\zeta}+\frac{v^{M}}{\Delta}\left[\delta_{1} m+\lambda \chi\left[E^{h} h-\mu\right]\right]
$$

\section{A.2 Proof of the outburst theorem}

Proof of (i): We know from (11) that tension falls in a neighborhood of $\left(0,-\delta_{1} m\right)$ for both the stress-prone and the stress-resistant individual (see fig. 2 for an illustration). If the growth rate of stress $\Phi$ is negative, the threshold level $W^{*}$ from (13) is negative as well and the individual never displays outbursts. Hence, a necessary condition for outbursts to occur is $\Phi>0$ which is (a) in the theorem.

By construction, $W(t) \in\left[0, \bar{W}\left[\right.\right.$. For modestly positive $\Phi$, it could result that $W^{*}>\bar{W}$ (see again fig. 2). Hence, we need $W^{*}<\bar{W}$ which is condition (b).

Proof of (ii): The necessary condition (a) to be a bad stabilizer reads with (12)

$$
\Phi>0 \Leftrightarrow \phi \frac{p}{a}>\delta_{0} .
$$

Condition (b) reads with (13)

$$
W^{*}<\bar{W} \Leftrightarrow \frac{\delta_{1} m}{\Phi}<\bar{W} \Leftrightarrow \Phi>\frac{\delta_{1} m}{\bar{W}}
$$


where the second "if and only if" used $\Phi>0$ from the necessary condition (a). Plugging in $\Phi=\phi \frac{p}{a}-\delta_{0}$ from (12), both necessary conditions are satisfied iff

$$
W^{*}<\bar{W} \text { and } \Phi>0 \Leftrightarrow \Phi>\frac{\delta_{1} m}{\bar{W}} \Leftrightarrow \phi \frac{p}{a}>\frac{\delta_{1} m}{\bar{W}}+\delta_{0}
$$

Proof of (iii): We understand the role of $W(t)$ by remembering that from (13) and (12), $W^{*}=\frac{\delta_{1} m}{\Phi}=\frac{\delta_{1} m}{\phi_{\frac{p}{a}}-\delta_{0}}$. For a given stress level $W(t)$, we can ask whether $W^{*}$ is larger or smaller than $W(t)$. Under equality, $W(t)=W^{*}=\frac{\delta_{1} m}{\phi \frac{p}{a}-\delta_{0}}$. Solving this for $\phi \frac{p}{a}$ in an attempt to draw this into fig. 3, we get

$$
\phi \frac{p}{a}=\frac{\delta_{1} m}{W(t)}+\delta_{0}
$$

\section{A.3 The frequency of outbursts}

Given the solution in (15), the length of time $T$ between two outbursts, i.e. how long it takes for an outburst to occur when the current stress level is $W(t)=\bar{W}-\Delta$, is

$$
\bar{W}=W^{*}+\left(\bar{W}-\Delta-W^{*}\right) e^{\Phi T} \Leftrightarrow T=\Phi^{-1} \ln \frac{\bar{W}-W^{*}}{\bar{W}-\Delta-W^{*}} .
$$

The inverse $T^{-1}$ is then the frequency of outbursts presented in prop. 4 .

For simplicity, we compute the comparative static properties of $T$. In prop. 4 , we report the comparative static properties of $T^{-1}$.

(a) The effect $d T / d \Phi<0$ is immediately visible.

(b) The average length of time between two outbursts rises in $W^{*}$,

$$
\frac{d T}{d W^{*}}>0 \Leftrightarrow \frac{d}{d W^{*}} \frac{\bar{W}-W^{*}}{\bar{W}-\Delta-W^{*}}=\frac{-\left(\bar{W}-\Delta-W^{*}\right)+\left(\bar{W}-W^{*}\right)}{\left(\bar{W}-\Delta-W^{*}\right)^{2}}=\frac{\Delta}{\left(\bar{W}-\Delta-W^{*}\right)^{2}}>0
$$

(c) We also see that

$$
\frac{d T}{d \bar{W}}<0 \Leftrightarrow \frac{d}{d \bar{W}} \frac{\bar{W}-W^{*}}{\bar{W}-\Delta-W^{*}}=\frac{\bar{W}-\Delta-W^{*}-\left(\bar{W}-W^{*}\right)}{\left(\bar{W}-\Delta-W^{*}\right)^{2}}=\frac{-\Delta}{\left(\bar{W}-\Delta-W^{*}\right)^{2}}<0
$$

(d) Further, as $\Phi$ is not a function of $\Delta$ (see (12))

$$
\frac{d T}{d \Delta}>0 \Leftrightarrow \frac{d}{d \Delta} \frac{\bar{W}-W^{*}}{\bar{W}-\Delta-W^{*}}>0 .
$$

To compute this, we need to observe that from (13) and (10)

$$
W^{*}=\frac{\delta_{1} m-\phi \frac{p}{a} \omega}{\phi \frac{p}{a}-\delta_{0}}=\frac{\delta_{1}\left[\frac{v^{M}}{\Delta} \frac{\delta_{1}}{v_{0}} \frac{1}{1+\zeta}\right]^{1 / \zeta}-\phi \frac{p}{a} \omega}{\phi \frac{\underline{p}}{a}-\delta_{0}} .
$$

The threshold $W^{*}$ is therefore a decreasing function in $\Delta$.

To take this into account, define $F\left(W^{*}(\Delta), \Delta\right) \equiv \frac{\bar{W}-W^{*}(\Delta)}{\bar{W}-\Delta-W^{*}(\Delta)}$. Then

$$
\frac{d T}{d \Delta}>0 \Leftrightarrow \frac{d}{d \Delta} F\left(W^{*}(\Delta), \Delta\right)=\frac{\partial F\left(W^{*}(\Delta), \Delta\right)}{\partial W^{*}(\Delta)} \frac{d W^{*}(\Delta)}{d \Delta}+\frac{\partial F\left(W^{*}(\Delta), \Delta\right)}{\partial \Delta}>0 .
$$

As $\frac{\partial F\left(W^{*}(\Delta), \Delta\right)}{\partial W^{*}(\Delta)}>0$ from (A.7) and $\frac{d W^{*}(\Delta)}{d \Delta}<0$ from (A.8) but $\frac{\partial}{\partial \Delta} F\left(W^{*}(\Delta), \Delta\right)>0$ (the "direct effect"), the sign is ambiguous.

The web appendix is available at www.waelde.com/pub. 


\section{References}

Ahlin, C., and M. Shintani (2007): "Menu costs and Markov inflation: A theoretical revision with new evidence," Journal of Monetary Economics, 54(3), 753-784.

Ali, S. N. (2011): "Learning Self-Control," Quarterly Journal of Economics, 126(2), 857-893.

Allen, L., R. K. McHugh, and D. Barlow (1998): Emotional disorders: A unified protocolpp. 216-249. Barlow, David H. (Ed), (2008), Clinical handbook of psychological disorders: A step-by-step treatment manual (4th ed.), New York, Guilford Press.

Anderson, C., and B. Bushman (2002): "Human Aggression," Annual Review of Psychology, $53(1), 27-51$.

Baddeley, A. D., and G. J. Hitch (1974): "Anxiety, Processing Efficiency, and Cognitive Performance," The psychology of learning and motivation: Advances in research and theory, 8, $47-89$.

Baker, M., J. Gruber, and K. Miligan (2008): "Universal Child Care, Maternal Labor Supply, and Family Well-Being," Journal of Political Economics, 116(4), 709-745.

Banerjee, A., and S. Mullainathan (2008): "Limited Attention and Income Distribution," American Economic Review: Papers \& Proceedings, 98(2), 489-493.

Baumeister, R., B. Bushman, and A. Stack (1999): "Catharsis, Aggression, and Persuasive Influence: Self-Fulfilling or Self-Defeating Prophecies?," Journal of Personality and Social Psychology, 76(3), 367-376.

Becker, G., and Y. Rubinstein (2011): "Fear and the Response to Terrorism: An Economic Analysis," mimeo.

Bell, D. E. (1985): "Disappointment in Decision Making under Uncertainty," Operations Research, 33(1), 1-27.

Benhabib, J., and A. Bisin (2005): "Modeling internal commitment mechanisms and selfcontrol: A neuroeconomics approach to consumption-saving decisions," Games and Economic Behavior, 52(2), 460-492.

Benjamin, D., O. Heffetz, M. Kimball, and A. Rees-Jones (2012): "What Do You Think Would Make You Happier? What Do You Think You Would Choose?," American Economic Review, 102(5), 2083-2110.

Bentham, J. (1789, 1970): An Introduction to the Principles of Morals and Legislation. Burns, J.H. and Hart, H.L.A. (eds), University of London, The Athlone Press.

Bentolila, S., and G. Bertola (1990): "Firing Costs and Labour Demand: How Bad Is Eurosclerosis?," Review of Economic Studies, 57(3), 381-402.

Bernheim, D., and A. Rangel (2004): "Addiction and Cue-Tiggered Decision Processes," American Economic Review, 94(5), 1558-90.

Bertola, G., and R. Caballero (1994): "Irreversibility and Aggregate Investment," Review of Economic Studies, 61(207), 223-246.

Bloch, F., and V. Rao (2002): "Terror as a Bargaining Instrument: A Case Study of Dowry Violence in Rural India," American Economic Review, 92(4), 1029-1043. 
Borghans, L., A. Duckworth, J. Heckman, and B. ter Weel (2008): "The Economics and Psychology of Personality Traits," Journal of Human Resources, 43(4), 972-1059.

Borghans, L., J. Heckman, B. Golsteyn, and H. Meijers (2009): "Gender Differences in Risk Aversion and Ambiguity Aversion," Journal of the European Economic Association, 7(2-3), 649-658.

Brocas, I., and J. D. Carrillo (2008): "The Brain as a Hierarchical Organization," American Economic Review, 98(4), 1312 - 1346.

Bushman, B., R. Baumeister, and C. Phillips (2001): "Do People Aggress to Improve Their Mood? Catharsis Beliefs, Affect Regulation Opportunity, and Aggressive Responding," Journal of Personality and Social Psychology, 81(1), 17-32.

Bushman, B. J. (2002): "Does Venting Anger Feed or Extinguish the Flame? Catharsis, Rumination, Distraction, Anger, and Aggressive Responding," Personality and Social Psychology Bulletin, 28, 724-731.

Camacho, A. (2008): "Stress and Birth Weight: Evidence from Terrorist Attacks," American Economic Review: Papers \& Proceedings, 98(2), 511-515.

Caplin, A., and J. Leahy (2001): "Psychological Expected Utility Theory And Anticipatory Feelings," Quarterly Journal of Economics, 116(1), 55-79.

- (2004): "The supply of information by a concerned expert," Economic Journal, 114(497), 487-505.

Caplin, A., and A. Schotter (2008): The Foundations of Positive and Normative Economics: A Hand Book. Oxford Scholarship Online.

Card, D., and G. Dahl (2011): "Family Violence and Footbal: The Effect of Unexpected Emotional Cues on Violent Behavior," Quarterly Journal of Economics, 126(1), 103-143.

Carver, C., and J. Connor-Smith (2010): "Personality and Coping," Annual Review of Psychology, 61(1), 679-704.

Chaiken, S., and Y. Trope (1999): Dual-process theories in social psychology. Guilford Press.

Chen, Y.-F., and M. Funke (2005): "Non-Wage Labour Costs, Policy Uncertainty And Labour Demand - A Theoretical Assessment," Scottish Journal of Political Economy, 52, 687-709.

Compte, O., and A. Postlewaite (2004): "Confidence-Enhanced Performance," American Economic Review, 94(5), 1536-1557.

Connor-Smith, J., B. Compas, M. Wadsworth, A. Harding Thomsen, and H. Saltzman (2000): "Responses to Stress in Adolescence: Measurement of Coping and Involuntary Stress Response," Journal of Consulting and Clinical Psychology, 68(6), 976-992.

Conti, G., S. Frühwirth-Schnatter, J. Heckman, and R. Piatek (2014): "Bayesian exploratory factor analysis," Journal of Econometrics, 183(1), 31-57.

DAK (2014): Gesundheitsreport 2014. Deutsche Angestellten Krankenkasse (DAK Forschung).

Derakshan, N., and M. Eysenck (2009): "Anxiety, Processing Efficiency, and Cognitive Performance," European Psychologist, 14(2), 168-176. 
Dixit, A. (1989): "Entry and Exit Decisions under Uncertainty," Journal of Political Economy, $97(3), 620-638$.

Dixit, A. K., and R. S. Pindyck (1994): Investment Under Uncertainty. Princeton University Press.

Dufwenberg, M. (2002): "Marital investments, time consistency and emotions," Journal of Economic Behavior \& Organization, 48, 57-69.

Ellsworth, P., and K. Scherer (2003): Appraisal Processes in Emotionpp. 572-595. Davidson, Richard J. and Scherer, Klaus R. and Goldsmith, H. Hill (Eds.) Handbook of affective sciences. Series in affective science, Oxford University Press.

Eysenck, M., and M. Calvo (1992): "Anxiety and Performance: The Processing Efficiency Theory," Cognition and Emotion, 6(6), 409-434.

Eysenck, M. W., N. Derakshan, R. Santos, and M. Calvo (2007): "Anxiety and Cognitive Performance: Attentional Control Theory," Emotion, 7(2), 336-353.

Fehr, E., and K. Hoff (2011): "Introduction: Tastes, Castes and Culture: The Influence of Society on Preferences," Economic Journal, 121(556), F396-F412.

Fontaine, J., K. Scherer, E. Roesch, and C. E. Phoebe (2007): "The World of Emotions Is Not Two-Dimensional," Psychological Science, 18(12), 1050-1057.

Fudenberg, D., and D. K. Levine (2006): "A Dual-Self Model of Impulse Control," American Economic Review, 96(5), 1449 - 1476.

_ (2012): "Timing and Self-Control," Econometrica, 80(1), 1-42.

Geanakoplos, J., D. Pearce, and E. Stacchetti (1989): "Psychological Games and Sequential Rationality," Games and Economic Behavior, 1(1), 60-79.

Gross, J. (1998): "The Emerging Field of Emotion Regulation: An Integrative Review," Review of General Psychology, 2(3), 271-299.

(2008): Emotion Regulation.pp. 497-512. Lewis, M. and Haviland-Jones, J. and Feldman Barrett, L. (eds.) Handbook of Emotion: New York, Guilford Press.

Gross, J., and L. Feldman Barrett (2011): "Emotion Generation and Emotion Regulation: One or Two Depends on Your Point of View," Emotion Review, 3(1), 8-16.

Gul, F. (1991): "A Theory of Disappointment Aversion,” Econometrica, 59(3), 667-86.

Harstad, R., and R. Selten (2013): "Bounded-Rationality Models: Tasks to Become Intellectually Competitive.," Journal of Economic Literature, 51(2), 496-511.

Haushofer, J., and E. Fehr (2014): "On the psychology of poverty," Science, 344(6186), 862-867.

Haushofer, J., and J. Shapiro (2013): "Household Response to Income Changes: Evidence from an Unconditional Cash Transfer Program in Kenya," mimeo MIT.

Heckman, J., J. Stixrud, and S. Urzua (2006): "The Effects of Cognitive and Noncognitive Abilities on Labor Market Outcomes and Social Behavior," Journal of Labor Economics, $24(3), 411-482$.

Helliwell, J., R. Layard, and J. Sachs (2013): World Happiness Report. United Nations. 
Hobson, C., J. Kamen, S. J., C. Nethercut, J. Tiedmann, and S. Wojnarowicz (1998): "Stressful Life Events - A Revision and Update of the Social Readjustment Rating-Scale," International journal of stress management, 5(1), 1-23.

Hoffmann, J., B. von Helversen, and J. Rieskamp (2013): "Deliberation's Blindsight: How Cognitive Load Can Improve Judgments," Psychological Science, 24(6), 869-879.

Holmes, T., and R. Rahe (1981): "The Social Readjustment Rating Scale," Journal of Psychosomatic Research, 11(4), 213-218.

John, O., L. Naumann, and C. Soto (2008): Paradigm Shift to the Integrative Big Five Trait Taxonomypp. 114-158. John, O-P. and Robins, R.W. and Pervin, L.A.(eds.) Handbook of Personality: Theory and Research, New York: Guilford Press.

Kahneman, D., and A. Tversky (1979): "Prospect Theory: An Analysis of Decision under Risk," Econometrica, 47(2), 263-291.

Kahneman, D., P. Wakker, and R. Sarin (1997): "Back to Bentham? Explorations of Experienced Utility," Quarterly Journal of Economics, 112(2), 375-406.

Kalisch, R., K. Wiech, H. D. Critchley, and R. J. Dolan (2006): "Levels of appraisal: A medial prefrontal role in high-level appraisal of emotional material," NeuroImage, 30, 1458-1466.

Kanner, A., J. Coyne, C. Schaefer, and R. Lazarus (1981): "Comparison of Two Modes of Stress Measurement: Daily Hassles and Uplifts Versus Major Life Events," Journal of Behavioral Medicine, 4(1), 1-39.

Karlsen, E., R. Dybdahl, and J. Vitterso (2006): "The possible benefits of difficulty: How stress can increase and decrease subjective well-being," Scandinavian Journal of Psychology, 47, 411-417.

Kőszegi, B. (2006): "Emotional Agency," Quarterly Journal of Economics, 121(1), 121-156.

Köszegi, B., and M. Rabin (2006): "A Model of Reference-Dependent Preferences," Quarterly Journal of Economics, 121(4), 1133-1165.

Kling, J., J. Liebman, and L. Katz (2007): "Experimental Analysis of Neighborhood Effects," Econometrica, 75(1), 83-119.

Kling, J., J. Ludwig, and L. Katz (2005): "Neighborhood Effects on Crime for Female and Male Youth: Evidence from a Randomized Housing Voucher Experiment," Quarterly Journal of Economics, 120(1), 87-130.

Krueger, A. B., and D. A. Schkade (2008): "The reliability of subjective well-being measures," Journal of Public Economics, 92, 1833-1845.

Kuppens, P., I. van Mechelen, and F. Rijmen (2008): "Toward Disentangling Sources of Individual Differences in Appraisal and Anger," Journal of Personality, 76(4), 969-1000.

Laibson, D. (1997): "Golden Eggs and Hyperbolic Discounting," Quarterly Journal of Economics, 112(2), 443-477.

Laibson, D. (2001): "A Cue-Theory of Consumption," Quarterly Journal of Economics, 116(1), $81-119$. 
Lazarus, R. (1993): "From Psychological Stress To The Emotions: A History of Changing Outlooks," The Annual Review of Psychology, 44, 1-21. York.

(1999): Stress and emotion: A new synthesis. Springer Publishing Company, New

Lazarus, R., and S. Folkman (1984): Stress, Appraisal and Coping. Springer Publishing Company, New York.

Lerner, J., and D. Keltner (2000): "Beyond valence: Toward a model of emotion-specific influences on judgement and choice," Cognition and Emotion, 14(4), 473-493.

Lewis, M. D. (2005): "Bridging emotion theory and neurobiology through dynamic modeling," Behavioral and Brain Sciences, 28(2), 169-194.

Loewenstein, G. (2000): "Emotions in Economic Theory and Economic Behavior," American Economic Review, 90(2), $426-432$.

Lohmann-Haislah, A. (2012): Stressreport Deutschland 2012. Bundesanstalt für Arbeitsschutz und Arbeitsmedizin.

Loomes, G., and R. Sugden (1982): "Regret Theory: An Alternative Theory of Rational Choice under Uncertainty," Economic Journal, 92(368), 805-24.

(1986): "Disappointment and Dynamic Consistency in Choice under Uncertainty," Review of Economic Studies, 53(2), 271-82.

Mani, A., S. Mullainathan, E. Shafir, and J. Zhao (2013): "Poverty Impedes Cognitive Function," Science, 341(6149), 976-980.

Meier, B., M. Robinson, and B. Wilkowski (2006): "Turning the Other Cheek Agreeableness and the Regulation of Aggression-Related Primes," Psychological Science, 17(2), 136-142.

Merton, R. C. (1975): "An Asymptotic Theory of Growth under Uncertainty," The Review of Economic Studies, 42(3), 375-393.

Moors, A., and K. R. Scherer (2012): The role of appraisal in emotionpp. 135-155. Robinson, M. and Watkins, E. and Harmon-Jones, E. (eds.) Handbook of cognition and emotion, Guilford Press, New York, USA.

Mullainathan, S. (2002): "A Memory-Based Model of Bounded Rationality," Quarterly Journal of Economics, 117(3), 735-774.

Mullainathan, S., and E. Shafir (2013): Scarcity: Why Having Too Little Means So Much. Time Books, Henry Holt \& Company LLC, New York.

Nakamura, J., and M. Csikszentmihalyi (2001): The Concept of Flowpp. 89-105. C.R. Snyder and Shane J. Lopez (Eds.) Handbook of Positive Psychology, Oxford University Press.

O'Donoghue, T., and M. Rabin (1999): "Doing It Now or Later," American Economic Review, $89(1), 103-124$. 160.

OECD (2014): Making Mental Health Count. OECD, Paris. 
Paas, F., A. Renkl, and J. Sweller (2003): "Cognitive Load Theory and Instructional Design: Recent Developments," Education Psychologist, 38(1), 1-4.

Piatek, R. (2010): "Bayesian Inference for Factor Structure Models via Gibbs Sampling," Discussion paper, University Konstanz, CMS Discussion Paper 2010-03.

Rabin, M. (2013a): "An Approach to Incorporating Psychology into Economics," American Economic Review, 103(3), 617-622.

(2013b): "Incorporating Limited Rationality into Economics," Journal of Economic Literature, 51(2), 528-543.

Rayo, L., and G. Becker (2007): "Evolutionary Efficiency and Happiness," Journal of Political Economy, 115(2), 302-337.

Roseman, I., and C. Smith (2001): Appraisal theory: Overview, assumptions, varieties, controversiespp. 3-19. K. R. Scherer, A. Schorr, \& T. Johnstone (Eds.) Appraisal processes in emotion: Theory, methods, research, New York: Oxford University Press.

Russell, J., and L. Feldman Barrett (1999): "Core Affect, Prototypical Emotional Episodes, and Other Things Called Emotion: Dissecting the Elephant," Journal of Personality and Social Psychology, 76(5), 805-819.

Scherer, K., and T. Brosch (2009): "Culture-Specific Appraisal Biases Contribute to Emotion Dispositions," European Journal of Personality, 23(3), 265-288.

Scherer, K., A. Schorr, and T. Johnstone (2001): Appraisal Processes in Emotion: Theory, Methods, Research (Series in Affective Science). Oxford University Press, USA.

Selye, H. (1976): Stress without distresspp. 137-146. G. Serban (ed.), Psychopathology of Human Adaptation.

(1982): History and present status of the stress concept.pp. 7-17. Goldberger, L. and S. Breznitz (eds.) Handbook of Stress: Theoretical and Clinical Aspects, New York: The Free Press.

Sherman, J., B. Gawronski, K. Gonsalkorale, K. Hugenberg, T. Allen, and C. Groom (2008): "The Self-Regulation of Automatic Associations and Behavioral Impulses," Psychological Review, 115(2), 314-335.

Shiv, B., and A. Fedorikhin (1999): "Heart and Mind in Conflict: The Interplay of Affect and Cognition in Consumer Decision Making," Journal of Consumer Research, 26, 278-292.

Shorey, R. C., J. Temple, J. Febres, H. Brasfield, A. E. Sherman, and G. L. Stuart (2012): "The Consequences of Perpetrating Psychological Aggression in Dating Relationships: A Descriptive Investigation," Journal of Interpersonal Violence, 27(15), 2980-2998.

Skinner, E., K. Edge, J. Altman, and H. Sherwood (2003): "Searching for the Structure of Coping: A Review and Critique of Category Systems for Classifying Ways of Coping," Psychological Bulletin, 129(2), 216-269.

Skinner, E., and M. J. Zimmer-Gembeck (2007): "The Development of Coping," Annual Review of Psychology, 58, 119-144.

Smith, E., and S. Kosslyn (2007): Working Memorypp. 239-279. Edward E. Smith and Stephen M. Kosslyn (Eds.) Cognitive Psychology: Mind and Brain, Pearson Prentice Hall. 
Specht, J., S. Schmukle, and B. Egloff (2011): "Stability and Change of Personality Across the Life Course: The Impact of Age and Major Life Events on Mean-Level and Rank-Order Stability of the Big Five," Journal of Personality and Social Psychology, 101(4), 862-882.

Stigler, G., and G. Becker (1977): "De Gustibus Non Est Disputandum," American Economic Review, 67(2), 76-90.

Stiglitz, J., A. Sen, and J.-P. Fitoussi (2008): "Report by the Commission on the Measurement of Economic Performance and Social Progress," http://www.stiglitz-senfitoussi.fr/documents/rapport_anglais.pdf.

Stokey, N. L. (2008): The Economics of Inaction: Stochastic Control Models with Fixed Costs. Princeton University Press.

Strack, F., and R. Deutsch (2004): "Reflective and Impulsive Determinants of Social Behavior," Personality and Social Psychology Review, 8(3), 220-247.

Straus, M. A., S. L. Hamby, S. Boney-McCoy, and D. B. Sugarman (1996): "The Revised Conflict Tactics Scales (CTS2)," Journal of Family Issues, 17(3), 283-316.

Strotz, R. (1955/56): "Myopia and Inconsistency in Dynamic Utility Maximization," Review of Economic Studies, 23(3), 165-180.

Sweller, J. (1988): "Cognitive Load During Problem Solving: Effects on Learning," Cognitive Science, 12(2), 257-285.

Taubman, W. (2003): "Did he bang it? Nikita Khrushchev and the shoe," New York Times, 26 July.

Tong, E. (2010): "Personality Influences in Appraisal-Emotion Relationships: The Role of Neuroticism," Journal of Personality, 78(2), 393-417.

Tur-Prats, A. (2015): "Family Types and Intimate-Partner Violence: A Historical Perspective," mimeo Universitat Pompeu Fabra.

Wälde, K. (2005): "Endogenous Growth Cycles,” International Economic Review, 46, 867-894. (2011): "Production technologies in stochastic continuous time models," Journal of Economic Dynamics and Control, 35, 616-622.

(2015): "Current emotion research in economics," Survey invited by Emotion Review. Available at www.waelde.com/pdf/WaeldeEmotionResearch.pdf.

Yaniv, G. (1995): "Burnout, absenteeism, and the overtime decision," Journal of Economic Psychology, 16(2), 297-309. 
Institut de Recherches Économiques et Sociales

Université catholique de Louvain

Place Montesquieu, 3

1348 Louvain-la-Neuve, Belgique 